\title{
POPOLI REGIONALI E DIRITTO ALL'AUTODETERMINAZIONE: STUDIO DEL «CASO VENETO»
}

\author{
Michelangelo De DonÀ \\ Profesor Ayudante Doctor \\ de Derecho de la Unión Europea \\ CIESL/UNIMED Milano \\ Università degli Studi di Pavia \\ miguel_73@libero.it \\ Daniele Trabucco \\ Profesor Ayudante Doctor \\ de Derecho Internacional \\ CIESL/UNIMED Milano \\ Università degli Studi di Padova \\ daniele.trabucco@unipd.it
}

\section{RESUMEN}

Se examina desde el punto de vista constitucional italiano la realidad concreta del caso véneto a partir de la combinación de la propia realidad jurídica nacional, el Derecho de la Unión Europea y el Derecho internacional.

Palabras clave: Unión Europea, Italia, Véneto, Venecia, Derecho internacional público, derecho a la autodeterminación, pueblos, naciones, Estados, unidad, indivisibilidad.

\section{ABSTRACT}

We look into the particular case of Italian Veneto from a constitutional point of view, starting from the combination of its own national legal reality, the European Union's Legal system and International Law.

Keywords: European Union, Italy, Veneto, Venice, International Public Law, right to self-determination, peoples, nations, states, unity, indivisibility.

\section{ZUSSAMENFASSUNG}

Aus der italienischen verfassungsrechtlichen Perspektive wird die konkrete Realität des Falls Veneto untersucht, unter Berücksichtigung der Kombination aus der eigenen nationalen rechtlichen Realität, dem Recht der Europäischen Union sowie dem Internationalen Recht.

Schlüsselwörter: Europäische Union, Italien, Veneto, Venedig, Völkerrecht, Recht auf Selbstbestimmung, Völker, Nationen, Staaten, Einheit, Unteilbarkeit. 
SUMARIO: I. LA DEFINIZIONE DI «POPOLO» NELL'ORDINAMENTO COSTITUZIONALE ITALIANO.--II. I «POPOLI REGIONALI»E LE SCELTE STATUTARIE. QUANDO TUTTO CAMBIA PERCHÉ NULLA CAMBI.III. L'ESPRESSIONE «POPOLI REGIONALI» TRA UNITÀ POLITICA E SOVRANITÀ. IL CONTRIBUTO DELLA GIURISPRUDENZA COSTITUZIONALE.-IV. I «POPOLI REGIONALI» TITOLARI DI UN DIRITTO ALL'AUTODETERMINAZIONE? IL «CASO» DELLA REGIONE DEL VENETO.-V. «POPOLI REGIONALI»E DIRITTO INTERNAZIONALE PUBBLICO.-VI. L'AUTODETERMINAZIONE DEI POPOLI ED IL PRINCIPIO DI INDIVISIBILITÀ DELLA REPUBBLICA.-VII. SEGUE: L'AUTODETERMINAZIONE DEI POPOLI E DIRITTO ALLA SECESSIONE.-VIII. SEGUE: L'AUTODETERMINAZIONE DEI POPOLI COME CONSUETUDINE INTERNAZIONALE DI IUS COGENS E LE SUE RICADUTE NELL'ORDINAMENTO REGIONALE.-IX. SEGUE: AUTODETERMINAZIONE DEI POPOLI E ART. 2 DELLA COSTITUZIONE.-X. SEGUE: PRINCIPIO DI INTEGRITA' TERRITORIALE E ART. 80 DELLA COSTITUZIONE.-XI. BIBLIOGRAFIA*.

\section{LA DEFINIZIONE DI «POPOLO» NELL'ORDINAMENTO COSTITUZIONALE ITALIANO}

Insieme al territorio, che si presenta quale sfera di validità dell'ordinamento giuridico statale, deve essere considerato anche l'elemento personale costituito da un gruppo di uomini che vivono stabilmente su di esso. A differenza del territorio, questo gruppo si presenta in una molteplicità di posizioni rispetto allo Stato. In primo luogo, affermare che uno Stato non può esistere senza un complesso di persone fisiche, significa che queste costituiscono il presupposto di fatto dello Stato. In secondo luogo, ed in questo caso possono ritenersi elemento dello Stato, esse sono legate al medesimo da un rapporto permanente e, in virtù di questo, capaci di determinare il modo d'essere dello Stato ${ }^{1}$.

Il collegamento tra la nozione di popolo e la nozione di convivenza territoriale non è però sufficiente ai fini della costruzione teoretica del concetto di popolo. Infatti, soltanto la comune, stabile e generale sottoposizione ad un potere effettivo ed indipendente «costituisce una qualsiasi collettività umana» in «popolo» propriamente detto ${ }^{2}$, non bastando quei fattori unificanti di natura etnica o razziale o culturale o territoriale che possono

* I paragrafi da 1 a 5 sono di Michelangelo DE DonÀ e i paragrafi da 6 a 10 sono di Daniele Trabucco.

${ }^{1}$ Cfr. C. Mortati, Istituzioni di Diritto pubblico, vol. I, Padova, CEDAM, 1969, p. 115.

${ }^{2}$ In questo senso V. CRISAFulli, Lezioni di Diritto Costituzionale. Introduzione al Diritto Costituzionale italiano, vol. I, Padova, CEDAM, 1970, p. 59. 
rinvenirsi senza che vi sia un'unità statale, oppure trascenderne l'ambito spaziale-territoriale di riferimento. Pertanto, solo l'affermarsi di un centro di potere sovrano promuove il sorgere di interessi comuni, anche laddove questi mancavano, e ne provoca comunque di ulteriori ${ }^{3}$.

Se dal piano teoretico passiamo poi a quello «dommatico», la definizione di popolo sarà determinata da ciascun ordinamento giuridico stata$\mathrm{le}^{4}$. A livello costituzionale, è stato osservato, esistono due filoni culturali che stanno alla base di due diverse idee di popolo e di sovranità popolare. Una prima identifica il popolo (sovrano) nella comunità ordinata di governanti e di governati ${ }^{5}$; la seconda vede il popolo come il complesso dei governati che si contrappone ai governanti ${ }^{6}$. Si tratta di concezioni che avevano animato il dibattito in seno all'Assemblea Costituente e che alla fine hanno trovato riflesso nel Testo costituzionale del 1948. In altri termini, per il primo indirizzo il popolo è quel soggetto idealmente unitario $^{7}$ capace di esprimere una propria volontà, per il secondo, invece, è il popolo reale, composto non solo di individui, ma anche di gruppi, associazioni, comunità territoriali ${ }^{8}$. Lo stesso art. 1 della Carta costituzionale vigente sottintende questa dicotomia ${ }^{9}$. Nell'affermare che «La sovranità appartiene al popolo» sia quoad titulum, sia quoad exercitium («che la esercita nelle forme...»), la Costituzione ha rifiutato una concezione del popolo solo come mero sottoposto ai comandi dell'apparato statale, che peraltro presuppone un'idea di sovranità (oggi superata) attribuibile unicamente alla persona giuridica dello Stato $^{10}$, accogliendo una nozione di popolo quale comunità organizzata dal diritto ${ }^{11} \mathrm{e}$ cioè in quanto si sia dato una propria Costituzione fuori della quale non vi è sovranità ma

${ }^{3}$ Sull'evoluzione del concetto stesso di popolo R. GatTI, Il popolo dei moderni. Breve saggio su una finzione, Brescia, La Scuola, 2014, pp. 5-89.

${ }^{4}$ Cfr. V. Crisafulli, Lezioni..., op. cit., p. 60.

5 F. RANGEON, «Intérêt général et démocratie», Cahiers de philosophie politique et juridique de l'Université de Caen, n. 2 (1982), p. 94.

${ }^{6}$ In relazione a questo secondo significato V. E. ORLando, Principi di diritto costituzionale, Firenze, G. Barbèra, 1917, p. 276.

7 Dove l'aspetto subiettivo dello Stato corrisponde alla sua natura obbiettiva: cfr. E. Tosato, Persona, Società intermedie e Stato. Saggi, Milano, Giuffrè, 1989, p. 72.

${ }^{8}$ Una concezione nella quale sono presenti interessi diversi e sentimenti talora contraddittori: in merito G. FERRARA, «Alcune osservazioni su popolo, Stato e sovranità nella Costituzione italiana», Rass. Dir. Pubbl., n. 2 (1965), pp. 272 e ss.

${ }^{9}$ Cfr. D. Nocilla voce «Popolo (dir. cost.)», Enc. dir., vol. XXXIV, Milano, Giuffrè, 1985.

${ }^{10}$ Cfr. E. CRosa, «Il principio della sovranità dello Stato nel diritto italiano», Arch. giur., s. IV, vol. XXV (1933), pp. 145 e ss.

${ }_{11}$ Sul punto D. NocILLA, voce «Popolo», op. cit. 
solo arbitrio popolare, una massa con le sue passioni e le sue debolezze ${ }^{12}$. La Costituzione stessa non è atto dello Stato-apparato che quest'ultimo abbia imposto al popolo dall'alto, bensì atto dello stesso popolo ${ }^{13}$, nato dal referendum istituzionale del 2 giugno 1946 ed elaborato da un'Assemblea costituente eletta a suffragio universale. In merito poi all'esatta identificazione dei criteri di appartenenza a questa comunità, l'intenzione dei Costituenti è stata quella di accettare implicitamente quanto era risultato prevalente nella dottrina costituzionalistica ${ }^{14}$ secondo la quale fanno parte del popolo solo quei soggetti che si pongono in un particolare rapporto con lo Stato, ossia il rapporto di cittadinanza ${ }^{15}$ (il c.d. status civitatis) attualmente normato dalla legge ordinaria dello Stato 05 febbraio 1992 n. 91. Non sembra che, oltre alla cittadinanza, siano richiesti altri requisiti affinché un soggetto faccia parte del popolo, diversamente da quanto avviene per il concetto di Nazione per la quale vengono in considerazioni vincoli di natura sociale, morale o naturale ${ }^{16}$, e neppure il raggiungimento di un numero minimo di soggetti ${ }^{17} \mathrm{o}$ il fatto che la comunità risieda stabilmente su di un territorio. In conclusione, quindi, la Carta del 1948 non pone alcun vincolo in capo al legislatore statale quanto ai criteri cui attenersi per le determinare le condizioni di acquisto e di perdita della cittadinanza salvo i limiti derivanti dal rispetto di altri principi costituziona$\operatorname{li}^{18}$ e dall'art. 22 Cost.

12 Così C. Esposito, «Commento all'art. 1 della Costituzione», in La Costituzione italiana. Saggi, Padova, CEDAM, 1954, pp. 6 e ss. In dottrina non sono mancate voci critiche per cui l'espressione giuridicamente unitaria del popolo, che ha consentito il superamento tra rex e regnum, o meglio tra soggetto sovrano e collettività governata, presuppone ancora traccia del dualismo sovranità-libertà, Stato-società. Questa si rinviene proprio nella concezione tradizionale dello Stato moderno: espressione unitaria del popolo da un lato, ma dall'altro pur sempre rappresentativo. Ora, sostenere che lo Stato è rappresentativo del popolo significa escludere quell'unità di cui sopra. Infatti, è possibile ragionare di Stato rappresentativo proprio nella misura in cui la collettività rappresentata non si identifichi con esso, ma gli sia estranea: così V. Crisafulli, Stato e popolo nella Costituzione italiana, in Studi per il primo decennale della Costituzione, t. II, Milano, Giuffrè, 1958, pp. 139 e ss.

13 Il popolo cioè non solo titolare della sovranità costituita, ma anche della sovranità costituente: così G. Guarino, Lezioni di Diritto pubblico, vol. I, Milano, Giuffrè, 1967, p. 74.

${ }^{14}$ Explurimis D. Donati, Elementi di diritto costituzionale, Padova, CEDAM, 1932, p. 20.

${ }^{15}$ Cfr. R. Romboli, «Problemi interpretativi della nozione giuridica di popolo», Riv. trim. dir. pubbl, vol. 34 (1984), pp. 163-164.

${ }^{16}$ In questo senso P. BisCaretTi di RufFia, voce «Nazione», in Nov. Dig. It., vol. IX, Torino, UTET, 1965, p. 184.

${ }^{17}$ In questo senso R. Romboli, «Problemi interpretativi...», op. cit., pp. 164-165. Contra G. DEL VeCCHIO, Lo Stato moderno ed i suoi problemi, Torino, Giappichelli, 1967, pp. 14-15.

18 Ancora, sul punto, R. Romboli, «Problemi interpretativi...», op. cit., p. 166. 


\section{I «POPOLI REGIONALI» E LE SCELTE STATUTARIE. QUANDO TUTTO CAMBIA PERCHÉ NULLA CAMBI}

Nello Statuto della Regione del Veneto, l'ultimo in ordine di tempo ad essere stato approvato dopo la riforma avviata dalla legge costituzionale 22 novembre 1999 n. 1, si trova un espresso riferimento al «popolo veneto». In particolare, nella legge regionale statutaria veneta 17 aprile 2012 n. 1, si riconosce nell'art. 2 il principio di autogoverno del popolo veneto (comma 1) nonché l'impegno da parte della Regione di salvaguardare e promuovere l'identità storica del popolo e della civiltà veneta (comma 2) ${ }^{19}$. Alla luce, dunque, delle considerazioni svolte nel paragrafo precedente, come deve essere valutato questo riferimento?

In primo luogo va osservato come, proprio con particolare riferimento alla Regione del Veneto, già il precedente Statuto del 1971 all'art. 1, comma 2, richiamava il concetto di autogoverno del popolo veneto. Anzi, la stessa formulazione della disposizione statutaria del 1971 è rimasta invariata nel nuovo. Inoltre, il Veneto non rappresenta un caso isolato. Oltre a richiami al «popolo sardo» ${ }^{20}$ ed al «popolo siciliano» ${ }^{21}$, adottati negli Statuti delle Regioni ad ordinamento differenziato rispettivamente di Sardegna e di Sicilia in materia di iniziativa legislativa, anche altre Regioni ad ordinamento comune, soprattutto dopo la 1. cost. n. 1/1999, hanno intrapreso questa strada ${ }^{22}$. Pur non utilizzando il termine popolo, alcuni Statuti si sono spinti a voler riconoscere e promuovere un rilievo ultraregionale, ed in alcuni casi ultranazionale, delle rispettive comunità regionali, introducendo parametri di ispirazione delle politiche regionali più ampi dell'effettivo interesse regionale ${ }^{23}$ : così ad esempio nello Statuto della Regione Abruzzo

\footnotetext{
${ }_{19}$ Recita l'art. 2 dello Statuto vigente della Regione del Veneto: «L'autogoverno del popolo veneto si attua in forme rispondenti alle caratteristiche e alle tradizioni della sua storia (comma 1). La Regione salvaguarda e promuove l'identità storica del popolo e della civiltà veneta e concorre alla valorizzazione delle singole comunità. Riconosce e tutela le minoranze presenti nel proprio territorio (comma 2)».

20 Art. 12 della legge costituzionale 26 febbraio 1948, n. 2 (Statuto speciale della Regione siciliana).

${ }^{21}$ Art. 28 della legge costituzionale 26 febbraio 1948, n. 3 (Statuto speciale della Regione Sardegna).

22 Sul punto A. Morrone, «Lo Statuto regionale, dopo le riforme», in A. Cariola, E. Castorina e A. Ciancio (a cura di), Studi in onore di Luigi Arcidiacono, Torino, Giappichelli, 2010, pp. 2237 e ss.

${ }_{23}$ Cfr. R. Dickman, «Popolo e popolazione nella Costituzione e negli Statuti», federalismi.it, n. 22 (2004), pp. 4-6.
} 
dove all'art. 1, comma 1, si prevede che «La Regione Abruzzo rappresenta la comunità dei cittadini, anche residenti all'estero, che per storia, tradizioni e cultura la costituiscono», oppure l'art. 2, comma 1, lett. g) dello Statuto della Regione Emilia-Romagna che tra i suoi obiettivi prioritari pone «il riconoscimento degli emiliano-romagnoli nel mondo».

In secondo luogo, se a prima vista l'espressione «popolo veneto» o, più in generale «popoli regionali», appare incoerente con la classica dottrina del diritto costituzionale che riserva alla parola popolo il significato di «elemento personale dello Stato» ${ }^{24}$, ad una più approfondita analisi si può constatare che questa non è l'unica lettura possibile. Già in altre Carte costituzionali, come quella tedesca, spagnola, svizzera e statunitense, il termine in questione è utilizzato con riferimento alle rispettive comunità regionali ${ }^{25}$. Questo, tuttavia, non significa, come invece è stato sostenuto, che il riferimento negli Statuti ai rispettivi popoli regionali non contiene nulla di eversivo, limitandosi solo a rimarcare la presenza di tratti identitari senza assumere alcun preciso valore giuridicamente rilevante $^{26}$. Proprio al fine di scongiurare inammissibili sviluppi delle politiche e dell'ordinamento regionale, non si può non assegnare alle disposizioni statutarie, contenenti i riferimenti ai «popoli regionali», «pieno valore giuridico a patto però di ricondurre il significato entro i confini dell'ordinamento costituzionale» ${ }^{27}$. In questo contesto, dunque, l'espressione popolo regionale non prescinde dal quadro costituzionale interno, assumendo il significato di una comunità titolare di diritto all'autogoverno, inteso quale valorizzazione dei caratteri culturali, sociali, identitari e sociali che già $i$ principi costituzionali sullo «Stato regionale» intendono garantire e sviluppare ${ }^{28}$. In altri termini, soprattutto con riferimento allo Statuto della Regione del Veneto, il concetto di autogoverno, da sempre utilizzato per indicare fenomeni di valorizzazione della democrazia dal basso ${ }^{29}$, è onto-

${ }^{24}$ Cfr. M. AinIs, «Il Veneto viene prima dell'Italia. Lo slogan che diventa Statuto», Corriere della Sera, 23 ottobre 2011.

${ }_{25}$ Così L. Antonini, «Una terra, un popolo, uno Statuto», Il Diritto della Regione, n. 3 (2011), e R. RombolI, voce «Popolo», Enc. giur. Treccani, vol. XXIII, Roma, 1991.

${ }^{26}$ Cfr. L. Benvenuti, «Lo Statuto del Veneto, e oltre», Il Diritto della Regione, n. 3 (2011).

${ }^{27}$ Le considerazioni sono di A. Morrone, «Postfazione: a proposito del carattere "veneto" dello Statuto regionale», in L. Benvenuti, G. Piperata e L. Vandelli (a cura di), Commento dello Statuto della Regione del Veneto, Venezia, Cafoscarina, 2012.

${ }^{28}$ Cfr. AAVV, Veneto. L'autonomia statutaria, a cura di M. MaLo, Torino, Giappichelli, 2012, pp. 100-101.

29 Sul punto M. Cammelli, voce «Autogoverno», in N. Bobbio, N. Matteucci e G. PasQUINO (dirs.), Dizionario di politica, Milano, Giuffrè, 1990, pp. 73 e ss. 
logicamente collegato a quello di «popolo veneto», risultando funzionale a proclamare e riconoscere «la relativa specificità in chiave prettamente identitaria e, per ciò solo, autonomistica» ${ }^{30}$.

\section{L'ESPRESSIONE «POPOLI REGIONALI» TRA UNITÀ POLITICA E SOVRANITÀ. IL CONTRIBUTO DELLA GIURISPRUDENZA COSTITUZIONALE}

Il contributo della giurisprudenza della Corte costituzionale sul punto è stato fondamentale. Il giudice delle leggi, soprattutto nella sentenza Corte cost. 14 novembre 2000 n. 496, aveva stabilito, sia pure con riferimento al procedimento di revisione della Carta costituzionale vigente ex art. 138 Cost, che il popolo è uno solo, quello «che dà forma all'unità politica della Nazione» ${ }^{31}$. Il richiamo al popolo evocato dalla Regione del Veneto nella delibera legislativa dell'08 ottobre 1998 (cui la sentenza n. 496/2000 si riferiva), chiamato a pronunciarsi in sede di referendum consultivo regionale sull'attribuzione alla Regione di forme e condizioni particolari di autonomia, è solo «una frazione autonoma (del corpo elettorale) insediata in una porzione del territorio nazionale» cui non era e non è consentito farsi portatore di modificazioni costituzionali «giacché le regole procedimentali e organizzative della revisione, che sono legate al concetto di unità e indivisibilità della Repubblica (art. 5 Cost.), non lasciano alcuno spazio a consultazioni popolari regionali che si pretendano manifestazione di autonomia.» ${ }^{32}$. La Corte, nel caso di specie, non contestava la facoltà per le Regioni ad ordinamento comune di farsi promotrici di iniziative volte alla modifica della Costituzione e ricollegabili al potere di iniziativa legislativa dei Consigli regionali in base a quanto espressamente previsto dall'art. 121, comma 2, Cost., quanto il tentativo da parte della Regione di affermare l'autodeterminazione del popolo veneto sia pure mediante un referendum consultivo regionale ad alta valenza politica ${ }^{33}$. Ora, se è vero

30 Cfr. G. Piperata, «Art. 2 Statuto della Regione del Veneto», in L. Benvenuti, G. PipeRATA e L. VANDElli (a cura di), Commento dello Statuto della Regione del Veneto, Venezia, Cafoscarina, 2012, p. 55. In merito alla concezione secondo la quale al fondo dell'idea di autonomia vi è sempre un principio di autogoverno sociale si veda la lezione di G. BERTI, «Art. 5 Cost.», in G. Branca (a cura di), Commentario della Costituzione, vol. I, Bologna, Il Mulino, 1975, pp. 287-288.

31 Punto 5 del cons. in dir. della sent. n. 496/2000 Corte cost.

32 Si veda il punto 5 del cons. in dir. della sent. n. 496/2000 Corte cost.

${ }^{3}$ Cfr. A. Morrone, «Avanti popolo... regionale!», Quad. Cost., n. 3 (2012), p. 616. Cri- 
che la Corte ritiene, riprendendo per certi versi l'orientamento già espresso in un suo precedente ${ }^{34}$, in particolare nella sentenza Corte cost. 24 novembre 1992 n. 47035, che la decisione sulla modificazione delle norme costituzionali è dall'art. 138 Cost. rimessa primariamente alla rappresentanza politico-parlamentare» e che «all'interno del procedimento di formazione delle leggi costituzionali il popolo interviene solo come istanza di freno, di conservazione e di garanzia, ovvero di conferma successiva, rispetto a una volontà parlamentare di revisione già perfetta, che, in assenza di un pronunciamento popolare, consolida comunque i propri effetti giuridici» ${ }^{36}$, è anche vero che questa ricostruzione non conduce ad una concezione esclusiva della sovranità ${ }^{37}$. Per Palazzo della Consulta, come indicato nella sentenza Corte cost. 12 aprile 2002 n. 106, è indubbio che le autonomie ter-

ticano la sentenza, in quanto il referendum consultivo regionale, non essendo atto avente effetto immediato e vincolante sull'ordine costituzionale e politico dello Stato, non era idoneo ad incidere sulla formazione della legge di revisione costituzionale, E. BALBONI, «Il referendum consultivo nello Stato-comunità: perché vietarlo?», Le Regioni, n. 1-2 (2001), p. 218, e A. RugGeRI, «Ancora in tema di referendum regionali consultivi e di teoria delle fonti», Le Regioni, n. 1-2 (2001), p. 229.

${ }^{34}$ Mentre nella sent. n. 470/1992 Corte cost. la Corte lasciava intendere che qualunque aggravio del procedimento statale nella fase dell'iniziativa sarebbe stato costituzionalmente illegittimo, nella pronuncia n. 496/2000 sembra limitare questo principio alle sole ipotesi di revisione costituzionale. Sulla lettura delle due sentenza si veda, recentemente, G. FERRAIUOLO, «La Corte costituzionale in tema di referendum consultivi regionali e processo politico: una esile linea argomentativa per un esito (in parte) prevedibile», federalismi.it, n. 20 (2015), pp. 3-6. L'autore ritiene che, mentre nella sentenza n. 470/1992 Corte cost. la declaratoria di illegittimità costituzionale si sofferma sull'alterazione della tipicità dell'iniziativa legislativa del Consiglio regionale, nella sent. n. 496/2000 Corte cost. la dichiarazione di illegittimità è riferita alla inconciliabilità del duplice momento referendario: quello previsto dalla delibera legislativa regionale e quello ex art. 138 Cost. Quest'ultimo aspetto era stato rilevato anche da S. BARTOLE, «Riforme federali e consultazioni referendarie regionali: un abbinamento discutibile», Giur. Cost., n. 6 (2000), pp. 3819 e ss.

35 Cfr. M. Dogliani e F. CAssella, «La "solitudine" del Parlamento nella decisione sulla forma dell'unità nazionale», Le Regioni, n. 5 (1993), p. 1305. La sentenza n. 470/1992 della Corte costituzionale si riferiva sempre ad una delibera legislativa della Regione del Veneto del 05 marzo 1992 recante «Referendum consultivo in merito alla presentazione di proposta di legge statale per la modifica di disposizioni costituzionali concernenti l'ordinamento delle Regioni».

36 Così il punto 4.2. del cons. in dir. della sent. n. 496/2000 Corte cost. Con la sentenza 25 giugno 2015 n. 118 Palazzo della Consulta sembra prendere le distanze da precedenti sue decisioni con le quali aveva ravvisato nell'indizione di referendum regionali consultivi in materia di autonomia regionale «il rischio di influire negativamente sull'ordine costituzionale e politico dello Stato» (sent. n. 470/1992 citando la precedente n. 256/1989): su quest'ultimo punto si veda S. BARTOLE, «Pretese venete di secessione e storica questione catalana, convergenze e divergenze fra Corte costituzionale italiana e Tribunale costituzionale spagnolo, anche con ripensamenti della giurisprudenza della prima», Giur. Cost., n. 3 (2015), p. 940.

${ }^{37}$ Così A. Morrone, «Avanti popolo... regionale!», op. cit., p. 616. 
ritoriali sono «partecipi dei processi di articolazione e diversificazione del potere politico» ${ }^{38}$, manifestando la «comune derivazione dal principio democratico e dalla sovranità popolare» ${ }^{39}$, ma ciò non comporta una confusione tra l'unità politica che fonda un popolo e le forme di espressione della sovranità nelle sue dimensioni territoriali ${ }^{40}$. Per questo motivo l'interesse generale di una comunità politica non può e non deve essere confuso con quello della popolazione residente in una frazione del territorio della Repubblica. Né sarebbe possibile, proprio in ragione di questa unità, far discendere dall'articolazione politico-amministrativa della Repubblica, una «ripartizione del popolo, inteso in senso di comunità generale, in improbabili sue frazioni». La Corte costituzionale, nella sentenza 19 maggio $2010 \mathrm{n}$. 170, ha ben chiara la caratteristica propria delle minoranze linguistiche quali «comunità necessariamente ristrette e differenziate» ${ }^{41} \mathrm{ma}$ pienamente inserite nella Repubblica, istituzione complessiva chiamata ad esprimere e tutelare elementi identitari sempre all'interno della vasta e composita comunità nazionale. Questo preclude, quindi, al legislatore regionale di rappresentare o raffigurare, sia pure implicitamente, la «propria» comunità in quanto tale solo perché riferita, sotto il profilo personale, all'ambito territoriale della propria competenza ${ }^{42}$.

Alla luce di queste considerazioni, l'esistenza di un «popolo regionale» che sia altro rispetto alla popolazione dei residenti all'interno di una determinata Regione rimanda ad una concezione dei rapporti tra Stato e Regione del tutto estranea al regionalismo previsto nel nostro sistema

${ }^{38}$ Punto 3 del cons. in dir. della sent. n. 106/2002 Corte cost.

39 Ibid.

40 Ancora A. MorRone, «Avanti popolo... regionale!», op. cit., p. 617. Per un commento alla sentenza n. 106/2002 della Corte costituzionale si rinvia al saggio di N. LuPO, «Dalla Corte costituzionale uno "stop" (definitivo) ai Parlamenti regionali. Nota a Corte cost. n. 106/2002», amministrazioneincammino.it, 10 luglio 2002. La tesi per la quale, secondo la nostra Costituzione, nel Parlamento si risolverebbe in sostanza la sovranità popolare senza che le autonomie territoriali concorrano a plasmarne l'essenza, non potrebbe essere condivisa nella sua assolutezza, sembrando necessario distinguere fra sovranità come potere supremo, che in un ordinamento unitario non può che spettare allo Stato e derivazione dalla volontà popolare che spetta sicuramente, in ogni ente a carattere rappresentativo, e dunque con organi elettivi, all'ente medesimo, in quanto esponenziale della comunità governata: sul punto F. CuOCOLO, «Parlamento nazionale e "parlamenti" regionali», Giur. Cost., n. 2 (2002), pp. 475-476.

${ }^{41}$ Si veda il punto 4 del cons. in dir. della sent. n. 170/2010 Corte cost. Sul punto, poi, cfr. P. LEWIS GETI, «Federalismo linguistico, tutela delle minoranze ed unità nazionale. Un nemis a l'é tròp e sent amis a basto nen. Nota a Corte cost. n. 170/2010», Rivista AIC, n. 4 (2010), p. 5.

42 Cfr. G. Delledonne, «La Corte costituzionale si pronuncia sulla "lingua piemontese": fra tutela delle minoranze linguistiche e incerti limiti di un "costituzionalismo regionale”», Le Regioni, n. 4 (2011), p. 724. 
costituzionale. Del resto, già attraverso il richiamo ai dati testuali della Costituzione, in particolare agli articoli 5, 114, 116 e 119, e alla giurisprudenza costituzionale era chiara ed inequivoca la posizione di autonomia e non di sovranità da parte delle Regioni ${ }^{43}$. Si tratterebbe di una contraddizione costituzionale insanabile ritenere lo Stato regionale contemporaneamente fonte di autonomia e di sovranità ${ }^{44}$. Quest'ultima rimanda, infatti, come è stato puntualizzato nella sentenza Corte cost. 07 novembre 2007 n. 365, ad un federalismo incompatibile con il grado di autonomia proprio degli ordinamenti regionali ${ }^{45}$. Seppure anche a livello regionale si è in presenza di meccanismi e forme di esercizio della sovranità, da questo non discendono necessariamente né la qualifica di popolo a quelle parti di porzioni del corpo elettorale insediato nel territorio della Regione ${ }^{46}$, né l'attribuzione a tutti gli organi istituzionali, che si alimentano dal circuito della rappresentanza, del medesimo status giuridico ${ }^{47}$. In realtà, ha osservato la dottrina ${ }^{48}$, anche per lo Stato dovrebbero valere le stesse conclusioni cui si è giunti per gli ordinamenti regionali. Infatti, titolare della sovranità è sempre il popolo, quale forma dell'unità politica della Nazione, dal momento che esso, come le Regioni e gli enti locali territoriali, è una tra le «forme» in cui la sovranità viene esercitata ${ }^{49}$.

${ }^{43}$ Cfr. B. Caravita di ToritTo, «Il tabù della sovranità e gli "istituti tipici di ordinamenti statuali di tipo federale in radice incompatibili con il grado di autonomia regionale attualmente assicurato nel nostro ordinamento"», federalismi.it, n. 22 (2007), pp. 1 e ss.

${ }^{44}$ Così ancora $\mathrm{A}$. MorRone, «Avanti popolo... regionale!», op. cit.

${ }^{45}$ Cfr. il punto 8 del cons. in dir della sent. n. 365/2007 Corte cost. In questa pronuncia la Corte costituzionale ha dichiarato l'illegittimità della legge della Regione speciale Sardegna, la n. 7/2006, istitutiva della Consulta di garanzia statutaria nella parte in cui configurava lo Statuto «fonte di autonomia e sovranità del popolo sardo».

${ }^{46}$ Questo aspetto già emergeva dalla sentenza n. 470/1992 Corte cost. In merito P. VERONESI, «Il concetto costituzionale di "unità nazionale" e le sue implicazioni», in A. Pugiotto (a cura di), Per una consapevole cultura costituzionale. Lezioni magistrali, Napoli, Jovene, 2013, p. 355.

47 Cfr. P. CARETTI, «La "sovranità" regionale come illusorio succedaneo di una "specialità" perduta: in margine alla sent. della Corte costituzionale n. 365/2007», Le Regioni, n. 1 (2008), p. 224. Contra O. CHESSA, «La resurrezione della sovranità statale nella sent. n. 365/2007», Le Regioni, n. 1 (2008), pp. 227-238.

${ }^{48}$ Cfr. S. Bartole, «La Corte costituzionale "chiude" al federalismo dal basso», Giur. Cost., n. 6 (2007), p. 4043.

${ }_{49}$ Così V. CRISAFulli, La sovranità popolare nella Costituzione italiana, in Scritti giuridici in memoria di V. E. Orlando, Padova, CEDAM, 1957, pp. 407 e ss. 


\section{I «POPOLI REGIONALI» TITOLARI DI UN DIRITTO ALL'AUTODETERMINAZIONE? IL «CASO» DELLA REGIONE DEL VENETO}

A prendere maggiormente sul serio il riferimento al proprio «popolo regionale» è stata la Regione del Veneto la quale, con la legge regionale 19 giugno $2014 \mathrm{n} .16^{50}$, ha introdotto la possibilità di un referendum consultivo sull'indipendenza della Regione. La legge in questione costituiva l'attuazione della risoluzione n. 44/2012 del Consiglio regionale, approvata poi con deliberazione n. 145/2012, con la quale si impegnava il Presidente del Consiglio medesimo ed il Presidente della Giunta regionale ad avviare urgentemente e con ogni risorsa a disposizione sia nell'ambito dell'Unione Europea, sia nell'ambito dell'ONU, ogni azione volta alla indizione di una consultazione referendaria funzionale all'accertamento della volontà del popolo veneto in ordine alla propria autodeterminazione. Il richiamo al principio internazionale di autodeterminazione, contenuto nella legge in esame, appare ancor più forzato, «perché se è vero che essa istituiva solo un referendum consultivo, è anche vero che il quesito che era posto agli elettori si muoveva drasticamente nei termini della secessione secca, della istituzione di una repubblica indipendente e sovrana, allontanandosi così, del tutto, non solo da quell'approccio graduale e dalla doverosa considerazione del complessivo quadro dei valori costituzionali (e tra questi di quelli supremi e irrinunziabili) ma escludendo dal suo orizzonte concettuale soluzioni diverse rispetto a quelle del distacco dallo Stato unitario» ${ }^{51}$.

Per rispondere alla domanda se il popolo veneto è titolare o meno di un diritto a decidere in merito alla propria autodeterminazione, è opportuno

\footnotetext{
${ }^{50}$ L'art. 1, comma 1, della legge veneta n. 16/2014 così prevedeva la formulazione del quesito: «Vuoi che il Veneto diventi una Repubblica indipendente e sovrana? Sì o no?». L'art. 4 della suddetta legge prevedeva la raccolta di un importo pari a 14 milioni di euro, a carico della collettività, per la celebrazione del referendum: una vera e propria condizione sospensiva. Su questo punto M. PIETRAngelo, «Qualche riflessione sui metodi della consultazione popolare al tempo di Internet, a margine dei referendum consultivi veneti su indipendenza ed autonomia», federalismi.it, n. 1 (2015), p. 6. Sul carattere centrifugo sia della legge regionale 14 giugno 2014, sia della legge veneta n. 16/2014, P. VIPIANA, «Le ripercussioni della crisi economica sull'assetto delle autonomie in Italia: un ritorno all'accentramento», in P. VIPIANA (a cura di), Tendenze centripete e centrifughe negli ordinamenti statali dell'Europa in crisi, Torino, Giappichelli, 2014, pp. 39-42.

${ }^{51}$ Cfr. G. Serges, «Autodeterminazione, diritto a decidere, indipendenza, sovranità (notazioni a margine della legge regionale del Veneto, n. 16 del 2014)», federalismi.it, n. 1 (2015), p. 11.
} 
svolgere alcune considerazioni di natura generale proprio sulla portata di questo diritto. Il principio di autodeterminazione è divenuto regola di diritto internazionale ${ }^{52}$ sia perché contenuto all'interno di alcune Convenzioni internazionali (ad esempio la Carta dell' ONU del $1945^{53}$ ed il Patto internazionale dei diritti civili e politici del $1966^{54}$ ), sia perché con il tempo ha acquistato il valore di norma consuetudinaria di ius $\operatorname{cogens}^{55}$ grazie ad una prassi che trova la sua base nella stessa Carta delle Nazioni Unite. L'autodeterminazione dei popoli è, però, una nozione della quale non è facile stabilirne l'esatto contenuto ${ }^{56}$. Essa, in linea generale, può essere definita come la libertà di un popolo di definire il proprio regime politico, economico e sociale, nonché la libertà di un popolo di accedere all'indipendenza come Stato separato oppure di staccarsi da uno Stato per aggregarsi a un altro ${ }^{57}$. La stessa Corte di Giustizia ha avuto occasione ${ }^{58}$ di specificarne meglio contenuto ed ambito di applicabilità: esso si applica soltanto ai popoli sottoposti a un Governo straniero, ossia ai popoli ancora soggetti a dominazione coloniale e alle popolazioni di territori conquistati e occupati con la forza ${ }^{59}$. Solo in questo caso, precisa la CIG, l'autodeterminazione si configura come un diritto dei popoli soggetti a dominio straniero di divenire indipendenti, di associarsi o integrarsi ad altro Stato indipendente. Inoltre, puntualizza sempre la Corte internazionale di Giustizia, salvo il caso dei territori coloniali, affinché il principio sia applicabile, non deve risalire oltre l'epoca in cui il principio stesso si è affermato come principio giuridico, ossia oltre l'epoca successiva alla fine della seconda guerra mondiale ${ }^{60}$.

52 Cfr. ibid., p. 7.

53 Art. 1, par. 2, e art. 55. La Carta delle Nazioni Unite è stata resa esecutiva in Italia con legge ordinaria dello Stato 17 agosto 1957 n. 848.

54 Art. 1. Il Patto internazionale dei diritti civili e politici è stato reso esecutivo con legge ordinaria dello Stato 25 ottobre 1977 n. 881.

55 Si veda il parere della CIG del 21 giugno 1971 sulla Namibia o il parere del 16 ottobre 1975 sul Sahara occidentale nonché, da ultimo, il parere del 22 luglio 2010 sul Kosovo.

56 Cfr. B. Conforti, Diritto Internazionale, Napoli, Jovene, 2015, p. 27.

57 G. Arangio-Ruiz, voce «Autodeterminazione (diritto dei popoli alla)», in Enc. giur. Treccani, vol. IV, Roma, 1988. Parla, invece, diritto di un popolo a vivere libero da qualsiasi tipo di oppressione, tanto interna che esterna, condizione questa prioritaria per il raggiungimento di relazioni amichevoli tra gli Stati membri e per un progresso economico dei popoli fondato su un'equa distribuzione delle risorse a livello sia internazionale che interno, F. LATTANZI, «Autodeterminazione dei popoli», in Digesto delle Discipline Pubblicistiche, vol. II, Torino, UTET, 1987, pp. 4 e ss.

58 Si vedano i pareri riportati in nota 55.

59 Il Consiglio di Sicurezza delle Nazioni Unite, con risoluzione n. 2625 (XXIV) del 1970, ha equiparato ai popoli soggetti a dominazione coloniale quelli vittime di segregazioni razziali o di regimi di appartheid (si pensi al caso del Sud Africa).

${ }^{60}$ Un approfondimento di questo punto si trova in M. BERTOLISSI, «Le iniziative 
Contro una siffatta ricostruzione del principio di autodeterminazione, una parte della dottrina, facendo leva sul parere del 22 luglio 2010 della Corte internazionale di Giustizia, relativo alla dichiarazione di indipendenza del Kosovo ${ }^{61}$, è pervenuta a diverse conclusioni. In particolare è stato posto l'accento sul punto in cui la Corte ritiene, nel paragrafo 70 del parere, che il principio di autodeterminazione valga anche al di fuori dei contesti precedentemente richiamati e che esso non contrasti con il principio di integrità territoriale, il quale è relegato unicamente ai rapporti tra Stati ${ }^{62}$ non applicandosi ai popoli ${ }^{63}$. Ne consegue, allora, come non solo il diritto internazionale non contenga alcuna proibizione ad una dichiarazione di indipendenza, ma che l'autodeterminazione stessa assuma un nuovo significato, ossia quale atto compiuto da un popolo che di propria iniziativa si autoriconosce e si autodefinisce tale e si autodetermina con un atto di volontà, esprimendo così un fatto che si palesa quale diritto ${ }^{64}$ anche indipendentemente dalle situazioni che fino ad oggi la stessa Corte internazionale di Giustizia aveva individuato ai fini dell'applicabilità del principio. La mancanza, quindi, di una definizione di populus a livello di diritto internazionale pubblico consentirebbe al «popolo veneto», per il solo fatto di considerarsi tale, di esercitare l'autodeterminazione. Né si potrebbe replicare che la situazione del Kosovo costituisce un caso a sé, dal momento che la specialità della vicenda kosovara è stata considerata dalla Corte né ostativa, né promotiva, ma semplicemente indifferente ${ }^{65}$.

In aggiunta a queste considerazioni, si è sostenuto in dottrina che, grazie al trasformatore permanente dell'art. 10, comma 1, della Carta costi-

della Regione Veneto per una maggiore autonomia e/o indipendenza», plebiscito2013.eu, pp. 1-7.

${ }^{61}$ Il 17 febbraio 2008, l'Assemblea del Kosovo con una votazione di 109 favorevoli su 120 membri totali (all'unanimità dei presenti, visti gli 11 assenti) approva la Dichiarazione di Indipendenza. Su richiesta della Serbia, il giorno successivo si è tenuta una pubblica riunione d'emergenza del Consiglio di Sicurezza dell'ONU (composto da 15 Stati, dei quali 5 permanenti). La Serbia è stata sentita e ha accusato la dichiarazione di indipendenza del Kosovo di essere un atto illegittimo, tanto ai fini del diritto interno quanto riguardo l'ordinamento internazionale. In conseguenza, l'Assemblea Generale dell'ONU con risoluzione 63/3 dell'8 ottobre 2008 ha usato il proprio potere di attivare la Corte Internazionale di Giustizia per chiederle un advisory opinion che non è una sentenza.

62 Paragrafo 80 del parere 22 luglio 2010 della CIG.

${ }^{63}$ Cfr., sul punto, A. Favaro, «Parere su "l'indizione della consultazione referendaria [...] al fine di accertare la volontà del Popolo Veneto in ordine alla propria autodeterminazione" (Risoluzione n. 44/2012 - Deliberazione n. 145 del Consiglio regionale del Veneto)», plebiscito2013.eu, p. 11.

${ }_{64}$ Ancora ibid., p. 7.

${ }^{65}$ In merito sempre ibid., p. 12. 


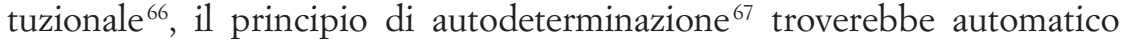
ingresso nel nostro ordinamento giuridico in quanto consuetudine internazionale ${ }^{68}$. Ora, trattandosi di una norma consuetudinaria di ius cogens, essa non solo si pone in posizione sovraordinata rispetto alle norme costituzionali (come del resto tutte le consuetudini internazionali), ma è dotata della caratteristica della inderogabilità rispetto ad altre consuetudini internazionali. Proprio in ragione di questa sua caratteristica, essa assumerebbe il rango di principio supremo della Costituzione ${ }^{69}$ con l'unica differenza che, mentre quest'ultimi sono immodificabili, le norme consuetudinarie di ius cogens potrebbero venir modificate unicamente da una consuetudine internazionale successiva ${ }^{70}$. In questo modo, l'equiparazione delle norme imperative consuetudinarie ai principi supremi immodificabili impedirebbe che, tramite un procedimento di revisione costituzionale, si possa violare una norma consuetudinaria di ius cogens, esponendo lo Stato italiano a illeciti particolarmente gravi sul piano internazionale ${ }^{71}$.

Da ultimo, alla tesi della indivisibilità della Repubblica di cui all'art. 5 della Costituzione, è stato opposto che se il termine «indivisibile» dovesse significare «intoccabile» o «immutabile», allora non si sarebbe potuto accettare come legittimo il Trattato di Osimo del 1975, né il precedente accordo dell'ottobre 1954 istituente le zone A e B del «Libero territorio di Trieste» (il c.d. Memorandum di Londra) ${ }^{72}$. Anzi, nell'esaminare il dettato costituzionale emergerebbe come già i costituenti avessero previsto la possibilità che il territorio della Repubblica subisse variazioni: l'art. 80 della Costituzione autorizza il Parlamento italiano a ratificare Trattati internazionali che «importino variazioni del territorio».

${ }^{66}$ Sul meccanismo di cui all'art. 10, comma 1, Cost., T. PERASSI, «La Costituzione italiana e l'ordinamento internazionale», in Scritti giuridici, Milano, Giuffrè, 1958, pp. 429 e ss.

${ }^{67}$ Nella sentenza del 1995, sull'affare riguardante Timor Est, la Corte internazionale di Giustizia definisce il diritto di autodeterminazione dei popoli come uno «dei principi essenziali del diritto internazionale contemporaneo», opponibile a tutti gli Stati della Comunità internazionale.

${ }^{68} \mathrm{Si}$ vedano, in merito, le interessanti considerazioni di L. AzZANO CANTARUTTI, «Elaborato per la commissione giuridica risoluzione 44», plebiscito2013.eu, pp. 2-6.

${ }_{69}$ La tesi è sostenuta da N. RonziTTI, «L'adattamento dell'ordinamento italiano alle norme imperative di diritto internazionale», Rass. parlam., n. 2/2002, pp. 503 e ss.

${ }^{70}$ Cfr. N. RonzitTi, Introduzione al Diritto Internazionale, Torino, Giappichelli, 2016, p. 254.

71 Sempre, a riguardo, ibid.

${ }^{72}$ Cfr. A. FAVARO, «Parere su "l'indizione della consultazione referendaria...», op. cit., p. 6. 


\section{V. «POPOLI REGIONALI»E DIRITTO INTERNAZIONALE PUBBLICO}

Le considerazioni fino ad ora condotte muovono dal presupposto che i veneti costituiscano un popolo cui è ricollegabile l'esercizio del diritto all'autodeterminazione. Si è già visto, nei paragrafi precedenti, come a livello di diritto interno non sia possibile parlare di «popolo veneto». Alla medesima conclusione si perviene anche qualora si guardi al diritto internazionale pubblico. In primo luogo, il popolo «non può mai considerarsi soggetto internazionale titolare del diritto di autodeterminazione» ${ }^{73}$, in quanto è difficile considerarlo quale ente unitario in grado di rapportarsi all'esterno sul piano delle relazioni internazionali, ma semmai può essere visto quale beneficiario del medesimo. Gli obblighi discendenti dal principio sono posti a carico dello Stato nazionale o di Stati terzi e solo da essi esigibili ${ }^{74}$ ed anche quando l'autodeterminazione si esplica verso l'interno, essa non altera il valore giuridico dell'obbligo di tutela esistente in capo agli Stati. È possibile ipotizzare, infatti, una categoria di obblighi erga omnes il cui scopo è quello di regolare l'esercizio dello sovranità dello Stato nel suo ambito territoriale ${ }^{75}$.

In secondo luogo, la mancanza di definizione a livello internazionale di popolo non rappresenta una carenza sul piano normativo, ma viceversa una precisa scelta che consente di adattarne la nozione alle articolazioni collettive esistenti all'interno di ciascuno Stato territoriale. Per individuare, quindi, l'esistenza di un «popolo veneto» ${ }^{76}$, all'interno dell'ordinamento costituzionale italiano, sarà necessario dimostrare che $\mathrm{i}$ veneti costituiscono un gruppo qualificato e distinto rispetto al resto degli italiani, al loro interno omogeneo, cui sia possibile riconnettere diritti che si riferiscono a beni giuridici irriducibilmente collettivi ${ }^{77}$ o riconoscere

${ }^{73}$ Cfr. F. Violi, «Autodeterminazione dei popoli e nuove forme di colonialismo», in M. Distefano (a cura di), Il principio di autodeterminazione dei popoli alla prova del nuovo millennio, Padova, CEDAM, 2014, p. 120.

${ }^{74}$ Sul punto ancora ibid.

75 Cfr. P. PiCONE, «Le reazioni collettive ad un illecito erga omnes in assenza di uno Stato individualmente leso», in P. PICONE (a cura di), Comunità internazionale e obblighi erga omnes, Napoli, Jovene, 2013, p. 629.

${ }^{76}$ Non si ravvisano le condizioni per l'applicazione delle categorie di minoranza nazionale o etnica. Sulla loro definizione F. Palermo e J. Woelk, Diritto Costituzionale comparato dei gruppi e delle minoranze, Padova, CEDAM, 2008, pp. 7-24.

77 Sempre F. Violi, «Autodeterminazione dei popoli...», op. cit., p. 121. 
l'esistenza di alcuni elementi presuntivi (identità della lingua, la comunanza di storia, tradizione e cultura, la localizzazione in un territorio ben preciso $)^{78}$. Solo attraverso lo spostamento verso una nozione meno monolitica di popolo ${ }^{79}$ ed il riconoscimento di uno spazio a tutti i gruppi portatori di identità specifiche i popoli potranno configurarsi come veri e propri soggetti del diritto internazionale e non solo come meri destinatari indiretti ${ }^{80}$, ma ad oggi l'ordinamento costituzionale italiano non ha optato per questa soluzione.

In terzo ed ultimo luogo, anche ritenendo che per l'individuazione del destinatario del principio in esame sia sufficiente la specifica volontà di autodeterminarsi ${ }^{81}$, resta aperto il problema della compatibilità degli strumenti giuridici volti ad accertarla con il principio di legalità costituzionale.

\section{L'AUTODETERMINAZIONE DEI POPOLI ED IL PRINCIPIO DI INDIVISIBILITÀ DELLA REPUBBLICA}

Nonostante la l. regionale n. 16/2014 abbia previsto una consultazione referendaria senza alcun vincolo giuridico né in capo agli organi della Regione del Veneto, né in capo agli organi dello Stato, non ci si poteva attendere una sentenza di rigetto da parte della Corte costituzionale chiamata a pronunciarsi sul ricorso in via d'azione del Governo della Repubblica n. 67/2014, promosso ai sensi dell' art. 127 Cost, che aveva impugnato tanto la legge n. 16/2014, quanto la legge regionale 19 giugno 2014 n. 15. Il giudice delle leggi, nella sentenza Corte cost. 25 giugno 2015 n. 118, dopo aver ribadito la sua giurisprudenza secondo la quale i referendum consultivi regionali non possono coinvolgere scelte di livel-

\footnotetext{
${ }^{78}$ Cfr. J. Crawford, «The Rights of Peoples: "Peoples" or "Governments"», in J. Crawford (a cura di), The Rights of Peoples, Oxford, Clarendon Press, 1988, p. 172.

79 Diverso il caso della Catalogna: la Costituzione spagnola fa riferimento ad una pluralità «pueblos» (preambolo) e «nacionalidades» (art. 2): cfr. G. FERRAIUOLO, «Due referendum non comparabili», Quad. Cost., n. 3 (2014), p. 705. Sulla trasformazione in termini giuridici delle diverse realtà nazionali presenti in Spagna cfr. M. HERRERO DE MiÑón, Memorias de estío, Madrid, Temas de Hoy, 1993, p. 123. Sulla possibilità di considerare i veneti un popolo si rinvia alla recenti e interessanti considerazioni di L. AzzANo CANTARUTTI, L'autodeterminazione del popolo veneto tra diritto internazionale e diritto interno, Lezione tenuta in data 12 maggio 2016 presso il Campus universitario Ciels, sede di Milano, paper.

${ }_{80}$ Cfr. E. PARIOTTI, I diritti umani: concetto, teoria, evoluzione, Padova, CEDAM, 2013, p. 166.

${ }^{81}$ Cfr. G. Palmisano, voce «Autodeterminazione dei popoli», Enc. dir., Annali, vol. V, Milano, Giuffrè, 2012, pp. 119 e ss.
} 
lo costituzionale ${ }^{82}$ salvo per i quesiti aventi ad oggetto l'iniziativa legislativa regionale ex art. 116, comma 3, Cost. ${ }^{83}$ poiché non preludono a sviluppi dell'autonomia eccedenti i limiti costituzionalmente previsti, ha chiarito come sia «giuridicamente erroneo equiparare il referendum consultivo a un qualsiasi spontaneo esercizio della libertà di manifestazione del pensiero da parte di più cittadini, coordinati tra loro. Il referendum è uno strumento di raccordo tra il popolo e le istituzioni rappresentative, tanto che si rivolge sempre all'intero corpo elettorale (o alla relativa frazione di esso, nel caso di referendum regionali), il quale è chiamato ad esprimersi su un quesito predeterminato. Inoltre, anche quando non produce effetti giuridici immediati sulle fonti del diritto, il referendum assolve alla funzione di avviare, influenzare o contrastare processi decisionali pubblici, per lo più di carattere normativo. Per questo, i referendum popolari, nazionali o regionali, anche quando di natura consultiva, sono istituti tipizzati e debbono svolgersi nelle forme e nei limiti previsti dalla Costituzione o stabiliti sulla base di essa» ${ }^{84}$. Palazzo della Consulta, in altri termini, chiarisce che, per quanto l'esito della consultazione sia quella di fornire un mero punto di vista, questo deve sempre inserirsi in una logica ed in una prospettiva di legalità costituzionale ${ }^{85}$ non potendo prescinderne. Con riferimento, dunque alla l. regionale n. 16/2014 non viene ritenuto illegittimo il ricorso allo strumento referendario, quanto piuttosto l'oggetto che si è inteso sottoporre alla consultazione popolare. Proponendo prospettive di secessione in vista della istituzione di un nuovo soggetto sovrano, il referendum consultivo veneto sull'indipendenza era incompatibile con i già sopra richiamati principi di unità ed indivisibilità della Repubblica ${ }^{86}$. Questi due termi-

${ }^{82}$ Punto 6 del cons. in dir. della sent. n. 118/2015 Corte cost.

${ }^{83}$ Con la sent. n. 118/2015 Palazzo della Consulta sembra, però, prendere le distanze da precedenti sue decisioni con le quali aveva ravvisato nell'indizione di referendum regionali consultivi, in materia di autonomia regionale, «il rischio di influire negativamente sull'ordine costituzionale e politico dello Stato» (sent. n. 470/1992 citando la precedente n. 256/1989): su questo punto si veda S. BARTOLE, «Pretese venete di secessione e storica questione catalana...», op. cit., p. 940.

${ }^{84}$ Punto 5 del cons. in dir. della sent. n. 118/2015 Corte cost.

${ }^{85}$ Riguardo al fatto che tutti i pubblici poteri devono essere guidati e limitati dalla Costituzione e dai vincoli da questa posti, si veda F. SALOMONI, «Legalità (costituzionale) e forma di Stato: aspetti teorici e profili partici di due concetti apparentemente in crisi», Riv. dir. cost. (2004), p. 111. Si veda, inoltre, anche il saggio di C. RUPERTO, «La legalità costituzionale», associazionedeicostituzionalisti.it, p. 2001.

${ }^{86}$ Così il punto 7.2. del cons. in dir. della sent. n. 118/2015 Corte cost. A riguardo F. CONTE, «La Corte costituzionale sui referendum per l'autonomia e l'indipendenza del Veneto. Non c'è due senza tre. Anche se»..., Quad. Cost., n. 3 (2015), p. 760. Analoghe considerazioni sono state espresse anche da C. ColaluCA, voce «Referendum regionali», in 
ni, che non possono essere letti e intesi come un'endiadi ${ }^{87}$, rappresentano rispettivamente un limite assoluto e un limite elastico. Infatti, se dall'indivisibilità si ricava il divieto ${ }^{88}$ di dividere o smembrare la Repubblica in più Stati indipendenti, anche da parte degli organi legislativi in sede costituente ${ }^{89}$, ovvero di procedere a secessione di una parte del suo territorio, dall'unità, proprio in virtù del suo carattere elastico, è stato possibile, soprattutto dopo la riforma del Titolo V nel 2001, modulare i rapporti tra Stato-Regioni-autonomie locali territoriali, superando quella concezione di unità politica affidata unicamente all'unità amministrativa dello Stato ${ }^{90}$ attraverso il concorso di tutte le persone giuridiche pubbliche costitutive della Repubblica ${ }^{91}$. Unico limite da rispettare consisterebbe, mediante lo spostamento delle competenze dal centro alla periferia, nell'evitare soluzioni tali da compromettere il carattere unitario e indivisibile (il corsivo è mio) dell'ordinamento ${ }^{92}$. In ragione di queste considerazioni non sarebbe neppure necessario richiamare la nota giurisprudenza costituzionale ${ }^{93}$ sui limiti impliciti alla revisione costituzionale, dal momento che le parole iniziali dell'art. 5 «fanno parte integrante del concetto di Repubblica, tutelato dagli artt. 1 e 139 Cost.» ${ }^{94}$. L'unico modo, dunque, con il quale sarebbe possibile procedere a una scissione di una parte del territorio nazionale, consisterebbe nel formarsi di una maggioranza parlamentare, rappresenta-

M. Malo e P. Santinello (a cura di), Veneto. Voci per un dizionario giuridico, Torino, Giappichelli, 2015, p. 194.

${ }^{87}$ A. PUBUSA, Sovranità popolare e autonomie locali nell'ordinamento costituzionale italiano, Milano, Giuffrè, 1983, pp. 166-167.

${ }^{88} \mathrm{Cfr}$. A. Amorth, La Costituzione italiana. Commento sistematico, Milano, Giuffrè, 1948, pp. 39-40, e R. Bifulco, «Art. 5 Cost», in R. Bifulco, A. Celotto e M. Olivetti (a cura di), Commentario alla Costituzione, t. I, Torino, Giappichelli, 2006, p. 140.

${ }^{89}$ Così C. Esposito, «Autonomie locali e decentramento amministrativo nell'art. 5 della Costituzione», in La Costituzione italiana. Saggi, Padova, CEDAM, 1954, p. 72.

${ }_{90}$ Cfr. M. Cammelli, «Amministrazione e (interpreti) davanti al nuovo Titolo V della Costituzione», Le Regioni, n. 6 (2001), pp. 1287-1288, e G. C. DE MARTIN, «L'amministrazione pubblica e la Costituzione», astrid-online.it, maggio 2006, p. 4.

${ }_{91}$ Sulla valenza del principio di unità ed indivisibilità della Repubblica anche dopo la riforma del Titolo, V C. BuZZACCHI, Uniformità e differenziazione nel sistema delle autonomie, Milano, Giuffrè, 2003, pp. 149-152.

${ }_{92}$ Cfr. A. D'AtenA, «La vicenda del regionalismo italiano ed i problemi della transizione al federalismo», in A. D'ATENA (a cura di), Federalismo e regionalismo in Europa, Milano, Giuffrè, 1994, p. 226.

${ }_{93}$ Corte cost. 29 dicembre 1988 n. 1146.

94 Si esprime così L. Paladin, Saggi di Storia costituzionale, a cura di S. Bartole, Bologna, Il Mulino, 2008, p. 176. Recentemente, rileggendo Livio Paladin, G. M. SALERno, «Principio di unità e ragionevole decentramento della Repubblica», in M. BERTOLISSI (a cura di), Riforme. Opinioni a confronto, Napoli, Jovene, 2015, p. 243. 
tiva di una volontà materialmente rivoluzionaria, in grado di trasformare il fatto in diritto per mezzo di fonti extra ordinem, là dove non bastassero o non fossero sufficienti le fonti legali di produzione del diritto ${ }^{95}$.

Non è mancato, tuttavia, il tentativo volto ad introdurre con un disegno di legge di revisione della Carta costituzionale il principio di autodeterminazione dei popoli tale da includere anche la facoltà di secessione. In particolare, in data 26 novembre 1996, è stato presentato al Senato della Repubblica il d.d.l. costituzionale n. 1803 che mirava ad introdurre, mediante modifica dell'art. 132 Cost., un diritto di distacco di parti del territorio nazionale in presenza di determinati requisiti. Per conseguire questo risultato l'art. 1 del d.d.l. in commento prevedeva che il primo periodo dell'art. 5 della Costituzione fosse così sostituito: «La Repubblica riconosce il principio di autodeterminazione dei popoli; rispetta e promuove le autonomie locali ${ }^{96}$. Se una siffatta modifica non avrebbe comportato problemi di compatibilità con il principio di unità, che trascende la consistenza attuale e futura delle parti integranti la Repubblica per cui lo Stato è e resta uno quale che sia la sua dimensione territoriale, altrettanto non può dirsi in rapporto al principio di indivisibilità ${ }^{97}$. Se quest'ultimo viene considerato in senso «prescrittivo», esso esclude qualsiasi separazione o secessione o perdita o diminuzione della consistenza territoriale della Repubbli$\mathrm{ca}$, se invece viene interpretato in senso relativo, ossia con riferimento ai possibili e tassativi modi ed alle forme prestabilite per una legittima divisibilità, allora sarebbe possibile bilanciare l'indivisibilità, quale attribuito della Repubblica, con il principio di autodeterminazione che contempli tra i suoi contenuti anche il diritto a secedere. Del resto, la stessa Carta costituzionale parrebbe confermare questo dato proprio nell'art. 80 che ragiona di Trattati internazionali che importino variazioni al territorio nazionale la cui ratifica sia autorizzata con legge ordinaria ${ }^{98}$. In attesa di ritornare in un prossimo paragrafo sul rapporto tra indivisibilità della Repubblica ed

${ }^{95}$ Cfr. B. Caravita di Toritto, «Federalismi, federalismo europeo, federalismo fiscale», federalismi.it, n. 9 (2011).

96 Cfr. F. Modugno, «Unità-indivisibilità della Repubblica e principio di autodeterminazione dei popoli (Riflessioni sull'ammissibilità-ricevibilità di un disegno di legge costituzionale comportante revisione degli artt. 5 e 132 Cost.)», in A. PACE (a cura di), Studi in onore di Leopoldo Elia, t. II, Milano, Giuffrè, 1999, p. 1012.

${ }_{97}$ Considera inscindibilmente connesse unità ed indivisibilità L. PaLAdIN, «Valori nazionali e principio di unità della Repubblica nella Costituzione italiana», in A. PACE (a cura di), Studi in onore di Mazziotti, Padova, CEDAM, 1995, p. 382.

${ }_{98}$ Sulla concezione «relativa» del principio di indivisibilità ancora F. Modugno, «Unità-indivisibilità della Repubblica e principio...», op. cit., pp. 1014-1016. 
art. 80 Cost., c'è da dire che un'eventuale introduzione in Costituzione del principio di autodeterminazione dei popoli non solo richiederebbe lo strumento formale della legge costituzionale, ma inciderebbe inevitabilmente su quei principi supremi che la Corte costituzionale, da ultimo con la sent. n. 118/2015, ha ritenuto insuperabili anche da una fonte di rango costituzionale $^{99}$. Questi principi, ha puntualizzato il giudice delle leggi, «non possono essere estremizzati fino alla frammentazione dell'ordinamento» ${ }^{100}$, mostrando in questo modo di aderire ad una concezione «proibitiva» della indivisibilità della Repubblica. Se è vero, infatti, «che la forma repubblicana non può essere oggetto di revisione, in forza dell'art. 139 Cost., anche i principi che la caratterizzano sono immodificabili» ${ }^{101}$.

\section{SEGUE: L'AUTODETERMINAZIONE DEI POPOLI E DIRITTO ALLA SECESSIONE}

Non appare del pari possibile dimostrare, con ragionevole fondamento, un diritto di autodeterminazione dei popoli applicabile agli abitanti della Regione del Veneto, e capace di inficiare il principio della indivisibilità della Repubblica sulla base del parere della Corte Internazionale di Giustizia del 22 luglio 2010 in merito alla indipendenza del Kosovo. Secondo la prevalente dottrina, come si è già avuto occasione di vedere, i requisiti di tale diritto sono essenzialmente due, la dominazione coloniale ovvero l'occupazione straniera con la forza. La CIG nel menzionato parere, su richiesta dell'Assemblea Generale dell'ONU cui a sua volta si era rivolta la Repubblica di Serbia, si è espressa sulla dichiarazione unilaterale di indipendenza da parte del Kosovo: in tale sede ha espressamente affermato come le dichiarazioni di indipendenza possono essere considerate lecite anche al di fuori dei due casi classici ricordati sopra (punto 79) ${ }^{102}$, e l'obbligo di rispettare l'integrità territoriale degli Stati esistenti incom-

99 F. Modugno, «Unità e indivisibilità della Repubblica come principio», in P. BIANCHI e C. Latini (a cura di), Costruire l'Italia. Dimensione storica e percorsi giuridici del principio di unità, Napoli, Jovene, 2013, p. 199.

100 Punto 7.2. del cons. in dir. della sent. n. 118/2015 Corte cost.

101 Cfr. G. Pistorio, «Il principio di unità ed indivisibilità della Repubblica nella giurisprudenza costituzionale», federalismi.it, n. 1 (2015), p. 8.

102 Sulla elasticità dell'autodeterminazione M. PERTILE, «Il parere sul Kosovo e l'autodeterminazione assente: quando la parsimonia non è una virtù», in L. GRADONI e E. MILANO (a cura di), Il parere della Corte internazionale di giustizia sulla dichiarazione di indipendenza del Kosovo: un'analisi critica, Padova, CEDAM, 2011, pp. 120-121. 
be solamente sugli Stati medesimi e non sui popoli (punto 80$)^{103}$. Il parere, peraltro, riconoscendo la forte disparità di vedute sulla ammissibilità di esercizio del diritto all'autodeterminazione dei popoli in casi che non vi rientrano, evita di entrare nel merito del dibattito sulla secessione-rimedio, limitandosi ad affermare che la dichiarazione unilaterale di indipendenza kosovara non viola il diritto internazionale pubblico (punto 83), ma non che sia da questo anche legittimata. Nel punto 56, infatti, la CIG prende le distanze dal parere della Corte Suprema del Canada sulla secessione del Québec ${ }^{104}$, presentato durante le udienze da molti Stati come il precedente giurisprudenziale più rilevante, precisando «che la domanda posta alla Corte Suprema Canadese riguardava l'esistenza di un diritto alla secessione, dovendo invece essa (la CIG) ricostruire l'eventuale esistenza di un divieto applicabile e, quindi, se la dichiarazione di indipendenza fosse stata adottata in violazione del diritto internazionale, non se tale dichiarazione fosse legittimata dal diritto internazionale ${ }^{105}$. Se nella decisione della Corte suprema del Canada del 1998 in relazione al caso del Québec, è stata affermata la legittimità di una secessione negoziata (e non unilaterale) con altri soggetti costituzionali ${ }^{106}$ attraverso ben precise condizioni procedurali e sostanziali sul presupposto che la «Costituzione non è una camicia di forza», questa opzione, ad avviso di chi scrive, deve essere comunque o espressamente consentita dal testo della Costituzione o ricavabile dai principi del sistema poiché, in caso contrario, si agirebbe extra ordinem ossia al di fuori del circuito del potere costituito. In tutti i casi, dunque, in cui la secessione non è negoziata, la comunità internazionale per decidere se una richiesta secessionista è legittima o meno non può non fare inevitabile riferimento all'ordinamento giuridico interno ${ }^{107}$. Va detto pure che il riferimento all'esercizio del diritto di autodeterminazione al di fuori dei due

${ }^{103}$ Cfr. F. Marcelli, «Il principio di autodeterminazione dei popoli nell'epoca del dominio della finanza», in M. Distefano (a cura di), Il principio di autodeterminazione dei popoli alla prova del nuovo millennio, Padova, CEDAM, 2014, p. 142.

${ }^{104} \mathrm{La}$ Corte suprema canadese, aggiungeva alle ipotesi classiche di diritto alla secessione, anche la «negazione dell'accesso al governo al fine di perseguire il proprio sviluppo politico, economico, culturale e sociale». In altri termini, una formulazione talmente ampia da consentire in molti, se non in tutti i casi, l'esercizio del diritto di secessione.

${ }^{105}$ Cfr. E. Milano, «Il parere consultivo della Corte internazionale di giustizia sulla dichiarazione di indipendenza del Kosovo: qualche istruzione per l'uso», sidi-isil.org, 2010, pp. 1 e ss.

${ }_{106}$ Cfr. T. E. FrosinI, «Costituzione, autodeterminazione, secessione», Rivista AIC, n. 1 (2015), p. 3.

107 Sul punto S. MANCINI, «Ai confini del diritto: una teoria democratica della secessione», Percorsi Costituzionali, n. 3 (2014), pp. 5-6. 
casi classici non è in realtà decisivo come a taluno possa sembrare. L'estensione del diritto ad altri casi, infatti, deve scontrarsi da un lato con la scarsità della prassi, dall'altro con l'assenza di una manifestazione di opinio iuris tale da giustificare una evoluzione del diritto di autodeterminazione in tal senso ${ }^{108}$. Il caso dell'indipendenza della Regione Veneto dall'Italia, comunque, non sembra rientrare in nessuno dei casi contemplati, non essendo né una colonia, né soggetta ad occupazione militare o a forme di oppressione che possano giustificare una secessione-rimedio unilaterale da uno Stato esistente. Non ha qualità analoghe di minoranza e, almeno fino$\mathrm{ra}$, non si è verificata una dichiarazione unilaterale di indipendenza seguita a una guerra come quella del Kosovo, che possa essere giustificata ex post almeno come non contraria al diritto internazionale.

Anche con riferimento alla dissoluzione dell'ex Repubblica socialista federale di Jugoslavia, essa (la dissoluzione), nonostante l'iniziale opposizione di Belgrado, fu considerata sempre come smembramento e mai come secessione, evitando, quindi, di dover far ricorso al principio di autodeterminazione dei popoli come norma di legittimazione da opporre al principio di integrità territoriale da parte delle autorità federali di Belgrado ${ }^{109}$. In questo modo è stato disinnescato il potenziale destabilizzante di un riconoscimento di un diritto all'autodeterminazione esterna in capo a qualsiasi componente nazionale o etnica che si volesse separare dal governo centra$\mathrm{le}^{110}$. Si può, quindi, ritenere che il diritto internazionale è sostanzialmente ostile alla secessione, intesa quale corollario del diritto di autodeterminazione, e la ammette non già come un diritto a sé stante bensì «come un diritto che scaturisce dalla lesione di altri diritti» ${ }^{111}$.

Alle conclusioni cui si è pervenuti nel corso di questo paragrafo, si potrebbe replicare che un conto è il principio di autodeterminazione dei popoli (principle of self determination), un altro il diritto all'autodeterminazione dei popoli (right of self-determination) cui fanno riferimento alcune

${ }^{108}$ In questo senso non è decisiva la dichiarazione di indipendenza della Repubblica autonoma di Crimea e di Sebastopoli datata 11 marzo 2014 che, nel preambolo, fa espresso riferimento alla dichiarazione di indipendenza del kosovara.

${ }^{109}$ Cfr. E. Milano, «L'autodeterminazione nei Balcani: soluzioni ad hoc per casi sui generis o consolidamento del principio nel contesto post-coloniale?», in M. DisTEFANO (a cura di), Il principio di autodeterminazione dei popoli alla prova del nuovo millennio, Padova, CEDAM, 2014, p. 40.

${ }^{110}$ M. CRAven, «The European Community Commission Arbitration on Yugoslavia», in British Yerabook of International Law, Oxford, Oxford University Press, 1996, p. 333, e D. RAIC, Statehood and the Law of Self-Determination, The Hague, Kluwer Law International, p. 2002.

${ }^{111}$ Cfr. S. Mancini, «Ai confini del diritto...», op. cit., pp. 1 e ss. 
convenzioni internazionali già ricordate. Mentre quest'ultimo costituisce un diritto-pretesa spettante ai popoli sottoposti a giogo coloniale, o più generalmente ai popoli sotto occupazione di forze straniere o ancora, secondo sviluppi recenti, a popoli che vivono in regime di congregazione razziale, il primo è un principio generale dell'ordinamento giuridico internazionale applicabile a tutti. Se il diritto all'autodeterminazione non sarebbe applicabile al nostro caso, non rientrando la Regione del Veneto nella «casistica» classica, i Veneti potrebbero però esercitare il diritto-libertà a proseguire l'obiettivo indipendenza (e, per la stessa ragione, anche catalani, scozzesi etc. ${ }^{112}$. In altre parole, l'autodeterminazione dei popoli si compone di «due distinti tronconi» corrispondenti alla bipartizione di cui sopra ${ }^{113}$. Questo modo di procedere appare, tuttavia, improntato ad un formalismo concettuale-normativo che non tiene conto dell'evoluzione dello stesso principio di autodeterminazione. Proprio a seguito della impetuosa affermazione dei movimenti indipendentisti nei contesti coloniali dell'Asia e dell'Africa, «il principio di autodeterminazione ha assunto i contorni di un vero e proprio diritto all'autodeterminazione» ${ }^{114}$, soprattutto al fine di circoscriverne il campo di applicazione. Anche aderendo alla tesi qui esposta, resta aperto il problema di quale sia l'esatto contenuto del principio di autodeterminazione. Si potrebbe rispondere che si è in presenza di un diritto-libertà funzionale all'ottenimento dell'indipendenza, tuttavia ci si dovrebbe chiedere in quali contesti esso è esercitabile dal momento che il principio così formulato è talmente ampio da consentire a tutti di attivarsi per questa finalità. Ora, ammesso pure che il principio di autodeterminazione (principle of self determination) consenta al Veneto di divenire uno Stato sovrano, è evidente come la sua natura di fonte consuetudinaria, avendo come conseguenza l'indipendenza della attuale Regione del Veneto dal resto dell'Italia, debba necessariamente «misurarsi» con l'ordinamento interno ed inevitabilmente scontrarsi con il limite dei principi supremi della Costituzione. La Corte, infatti, come avvenuto anche recentemente con la sentenza 22 ottobre 2014 n. 238 , ha utilizzato l'arma dei controlimiti in ipotesi di consuetudine internazionale che si ponga in contrasto con i suddetti principi.

112 Sul duplice significato dell'autodeterminazione si vedano, da ultime, le considerazioni di A. MocelLIN, «In merito al referendum per l'indipendenza del Veneto», leggioggi.it, 17 aprile 2015, pp. 1 e ss.

113 Ad esempio A. Cassese, Self-Determination of Peoples. A Legal Reappraisal, Cambridge, Cambridge University Press, 1995.

${ }_{114}$ E. Milano, voce «Autodeterminazione dei popoli», Diritto online. Approfondimenti Enciclopedici. Diritto Internazionale e Comparato, 2014, pp. 1-8. 


\section{SEGUE: L'AUTODETERMINAZIONE DEI POPOLI COME CONSUETUDINE INTERNAZIONALE DI IUS COGENS E LE SUE RICADUTE NELL'ORDINAMENTO REGIONALE}

A partire dalla sentenza della Corte cost. 18 giugno 1979 n. 48 il giudice costituzionale aveva affermato come l'intero meccanismo di cui all'art. 10, comma 1, Cost., pur assicurando la prevalenza della fonte consuetudinaria internazionale sulle norme costituzionali ${ }^{115}$, non poteva e non può «in alcun modo consentire la violazione dei principi fondamentali del nostro ordinamento costituzionale, tra i quali sono annoverati quelli di unità ed indivisibilità della Repubblica, operando in «un sistema costituzionale che ha i suoi cardini nella sovranità popolare e nella rigidità della Costituzione» ${ }^{116}$. In questo modo, si lasciava intendere che la distinzione tra consuetudini internazionali dotate di imperatività e consuetudini prive di questa caratteristica era ed è irrilevante in termini di recepimento nel diritto interno, costituendo una qualità incidente unicamente nei rapporti tra consuetudini internazionali. La recente sentenza n. 238/2014 ha sviluppato e meglio precisato l'impianto argomentativo della pronuncia del 1979. La questione sottesa alla sentenza n. 238/2014 concerneva, com'è noto, la consuetudine internazionale dell'immunità degli Stati dalla giurisdizione civile nella parte in cui copre gli atti iure imperii integranti crimini di guerra lesivi di diritti inviolabili quali, secondo il giudice remittente, l'art. 2 e l'art. 24 della Costituzione. Non è questa la sede per ripercorrere le vicende cui si riferisce la pronuncia della Corte costituzionale in esame. Quello che rileva, ai fini del presente lavoro, consiste nel fatto che Palazzo della Consulta riserva a sé l'esclusivo controllo dei controlimiti in ipotesi di norma consuetudinaria internazionale contrastante con i principi supremi dell'ordinamento al pari di quello che avviene con il diritto dell'Unione Europea. Il ragionamento dei giudici della legittimità costituzionale, almeno secondo una parte della dottrina, pare ritenere che anche se la consuetudine internazionale varca il confine grazie all'art. 10, comma 1 , Cost., disposizione che comporta un adeguamento automatico ma incapace di operare distinzioni, essa permane solo il tempo necessario a rendere accertabile dalla Corte la sua contrarietà ai principi fondamentali per

115 Così B. Conforti, Diritto..., op. cit., pp. 349-350.

116 Punto 3 del cons. in dir. della sent. n. 48/1979 Corte cost. 
essere infine «espatriata» prima ancora di potersi dichiarare illegittima la norma ${ }^{117}$ di adattamento ${ }^{118}$. Durante la sua «permanenza», alla luce di alcuni passaggi della Corte, la consuetudine sarebbe inapplicabile e perciò stesso improduttiva di effetti giuridici ${ }^{119}$ all'interno dell'ordinamento italiano e, dunque, anche all'interno dei singoli ordinamenti regionali. La Corte costituzionale, dunque, non solo respinge la tesi che equiparava le norme consuetudinarie di ius cogens ai principi supremi dell'ordinamento costituzionale ${ }^{120}$, ma si spinge a promuovere in modo esplicito un mutamento del diritto internazionale generale ${ }^{121}$. Palazzo della Consulta, con la sentenza n. 238/2014, delineando chiaramente una concezione non assolutistica dei principi supremi dell'ordinamento costituzionale, ammettendone la sottoposizione a bilanciamento, stabilisce però che questo è possibile a condizione che non ne sia «ridotto al minimo il sacrificio» ${ }^{122}$. Se il principio di autodeterminazione dei popoli, declinato come possibilità di secessione, venisse ritenuto principio supremo e bilanciato con l'art. 5 Cost., il secondo verrebbe sacrificato integralmente poiché, se anche una sola Regione italiana accedesse all'indipendenza dal resto dell'Italia, ver-

117 Legge l'art. 10, comma 1, Cost. quale norma sulla produzione per cui, in presenza di una consuetudine internazionale, si assisterebbe all'istantanea formazione di una norma di diritto italiano A. CASSESE, «Art. 10 Cost»., in G. BRANCA (a cura di), Commentario della Costituzione, Bologna, Il Mulino, 1975, p. 498. La Corte costituzionale, con la sent. n. 238/2014, pare aderire a questa tesi nel momento in cui, nel dispositivo della pronuncia, parla di «norma prodotta nel nostro ordinamento mediante recepimento» della consuetudine internazionale.

${ }^{118}$ Cfr. S. LEONE, «La sentenza n. 238/2014: una soluzione preordinata ad accentrare il sindacato sulle consuetudini internazionali», Quad. Cost., n. 4 (2014), p. 905. Ritiene, invece, che in ipotesi di conflitto tra una consuetudine internazionale ed un principio supremo della Costituzione non si attivi il trasformatore permanente dell'art. 10, comma 1, Cost. G. ZAGREBELSKI, Il sistema delle fonti del diritto, Torino, Giappichelli, 1984, p. 122. Precisa che il richiamo operato dall'art. 10, comma 1, della Carta al diritto internazionale consuetudinario non opera qualora quest'ultimo si ponga in contrasto «con i principi supremi dell'ordinamento costituzionale e i diritti inalienabili della persona» F. VARI, «La sentenza n. 238 del 2014: ancora sulla responsabilità degli Stati», Quad. Cost., n. 1 (2015), p. 168.

119 A questa conclusione giungono A. RUGGERI, «La Corte aziona l'arma dei "controlimiti" e, facendo un uso alquanto singolare delle categorie processuali, sbarra le porte all'ingresso in ambito interno di norma internazionale consuetudinaria», in «Itinerari» di una ricerca sul sistema delle fonti. Studi dell'anno 2014, vol. XVIII, Torino, Giappichelli, 2015, pp. 531 e ss., e S. LEONE, «La sentenza n. 238/2014...», op. cit., p. 904.

${ }_{120}$ Cfr. N. RonzitTi, Introduzione..., op. cit., p. 254.

${ }^{121}$ Cfr. L. Gradoni, «La sentenza n. 238/2014 della Corte costituzionale: Corte costituzionale italiana "controvento" sull'immunità giurisdizionale degli Stati stranieri?», Quad. Cost., n. 4 (2014), p. 906.

${ }^{122}$ Si veda il punto 3.1. del cons. in dir. della sent. n. 238/2014 Corte cost. Sul punto si veda il contributo di P. FARAGUNA, «La sentenza n. 238 del 2014: i controlimiti in azione», Quad. Cost., n. 4 (2014), pp. 900-901. 
rebbe leso in radice quel sintagma unità-indivisibilità da intendersi non quale «riflesso della integrità necessaria del territorio, né come argine alla pulsione autonomistica, bensì come sfera intima identitaria dell'ordinamento repubblicano» ${ }^{123}$ che è composta da tutti i territori che formano le Regioni elencate nell'art. 131 Cost. Inoltre, l'ingresso del principio di autodeterminazione ${ }^{124}$, avente come contenuto la possibilità di secessione, legittimerebbe l'esercizio di un nuovo potere costituente ${ }^{125}$ esauritosi con l'approvazione della Costituzione repubblicana il 22 dicembre $1947^{126}$ e ponendosi a fondamento di un nuovo ordinamento giuridico. Si darebbe, infatti, ingresso nell'ordinamento a una norma avente come fine, anche solo potenziale, quello di pervenire a un ordinamento indipendente rispetto a quello originario ed in questo modo sarebbero travolti i soggetti e le condizioni stesse del patto costituzionale.

Ancora più stringente risulterebbe il limite dell'unità-indivisibilità ove si volesse ritenere che il principio di autodeterminazione sia da considerare come vigente in quanto contenuto nei trattati internazionali ratificati e resi esecutivi nel diritto interno, perché la garanzia di osservanza degli obblighi internazionali contenuta nell'art. 117, comma 1, Cost. non consente che un obbligo internazionale collida con un principio costituzionale, tanto più quando si tratta di un principio supremo ${ }^{127}$, come del resto indicato dalla Corte costituzionale nelle sentenze «gemelle» 24 ottobre 2007 n. 348 e 24 ottobre 2007 n. 349.

In conclusione, quindi, il principio di autodeterminazione dei popoli, in quanto norma consuetudinaria internazionale, entra nell'ordinamento costituzionale italiano, ai sensi dell'art. 10 , comma 1, Cost., a patto però

${ }^{123}$ Cfr. P. Carnevale, «Unità della Repubblica ed unità del diritto oggettivo. Notazioni introduttive sulla Costituzione come fattore unitario nelle dinamiche della produzione normativa», federalismi.it, n. 1 (2015), p. 5.

${ }^{124}$ La Costituzione esclude che i principi ultimi dell'ordinamento dipendano e siano messi in discussione (il corsivo è mio) da decisioni di altre fonti: cfr., sul punto, S. BARTOLE, Interpretazioni e trasformazioni della Costituzione repubblicana, Bologna, Il Mulino, 2004, pp. 436-437.

125 Sulle definizioni di potere costituente si rinvia al lavoro di P. G. Grasso, Il potere costituente, Torino, Giappichelli, 2006, pp. 3-61.

126 Riguardo alla tesi per la quale la rinnovazione del potere costituente comporterebbe la rottura della continuità costituzionale preesistente: cfr. L. PALADIN, Le fonti del diritto italiano, Bologna, Il Mulino, 1996, p. 152, e M. Fioravanti, «Potere costituente e Diritto Pubblico», in P. Pombeni (a cura di), Potere costituente e riforme costituzionali, Bologna, Il Mulino, 1992, pp. 55 e ss. La stessa Corte costituzionale, nella sentenza 02 febbraio 1991 n. 47, si rifà a questa tesi secondo la quale la Costituzione si esaurisce in un unico atto di esercizio e non è più reiterabile (punto 3.1. del cons. in dir.).

${ }^{127}$ Cfr. G. SERGES, «Autodeterminazione...», op. cit., pp. 8-9. 
che non contempli tra i suoi contenuti la possibilità di scardinare il principio supremo di indivisibilità della Repubblica.

\section{SEGUE: AUTODETERMINAZIONE DEI POPOLI E ART. 2 DELLA COSTITUZIONE}

Pure l'affermazione circa il carattere precostituzionale del diritto di autodeterminazione dei popoli, che «entrerebbe» nell'ordinamento italiano in virtù del riconoscimento dei diritti inviolabili di cui all'art. 2 Cost., pare poco convincente. In primo luogo, anche ritenendo l'art. 2 una norma a fattispecie aperta, riferibile cioè alla tutela di valori e di diritti non espressamente previsti dalla Costituzione formale, il riconoscimento del diritto di autodeterminazione dei popoli, come degli altri diritti garantiti sul piano internazionale, non ne comporta una azionabilità sic et simpliciter nell'ordinamento interno, ma richiede sempre il completamento reciproco nell'interpretazione con le altre norme costituzionali ${ }^{128}$, o meglio una relazione di compatibilità dialettica con il dettato della Carta. Questo significa che il riconoscimento non può mai porsi in violazione dei limiti impliciti alla revisione costituzionale, tra cui il principio supremo della «Repubblica una e indivisibile» sancito dall'art. 5. Appare, in secondo luogo, non fondato sostenere che la pre-costituzionalità del diritto di autodeterminazione avrebbe avuto come conseguenza l'incompetenza funzionale della Corte costituzionale a decidere sulla l. veneta n. 16/2014. Come ha osservato la dottrina ${ }^{129}$, tutti i diritti hanno una componente regolativo-prestazionale, richiedono cioè discipline legislative complesse e, come tali, inevitabilmente sindacabili dalla Corte ai sensi dell'art. 134 Cost. La conferma di questo ci viene proprio dalla stessa 1 . regionale in esame, atto-fonte necessario non solo per la previsione/istituzione del referendum consultivo regionale, ma anche per affidare al presidente della Giunta regionale e a quello del Consiglio regionale la tutela in ogni sede competente, nazionale ed internazionale, del diritto del «Popolo Veneto» alla autodeterminazione ${ }^{130}$, in esecuzione della risoluzione n. 44/2012 approvata con deliberazione consiliare n. 145 del 28 novembre 2012 .

${ }^{128}$ Corte cost. 22 ottobre 1999 n. 388.

${ }^{129}$ Cfr. A. D’AloiA, «Introduzione. I diritti come immagini in movimento: tra norma e cultura costituzionale», in A. D'Alora (a cura di), Diritti e Costituzione. Profili evolutivi e dimensioni inedite, Milano, Giuffrè, 2003, pp. VII e ss.

${ }_{130}$ Art. 3, comma 2, della legge regionale veneta n. 16/2014. 


\section{SEGUE: PRINCIPIO DI INTEGRITA' TERRITORIALE E ART. 80 DELLA COSTITUZIONE}

Un'ultima argomentazione, posta a sostegno della legittimità del referendum consultivo regionale sull'indipendenza, era data dalla presunta violazione dell'art. 5 della Costituzione da parte del Trattato di Osimo del 10 novembre 1975, reso esecutivo con la legge ordinaria dello Stato 14 marzo 1977 n. 73. Secondo una parte della dottrina, quando il Parlamento italiano ha autorizzato la ratifica del Tratto tra l'Italia e la Repubblica socialista federale di Jugoslavia ha proceduto alla cessione di parti del territorio nazionale a uno Stato estero proprio in violazione della norma costituzionale sopra richiamata ${ }^{131}$.

Ora, anche volendo aderire alla tesi sopra prospetta, che confonde il piano del principio di integrità territoriale dell'art. 5 Cost. il quale opera sul versante esclusivamente interno, con la possibilità per lo Stato di stipulare trattati internazionali che importano variazioni del territorio ex art. 80 Cost., l'Accordo di Osimo non ha comportato alcuna modifica di tipo territoriale. Per comprenderne la ragione è opportuna e doverosa una breve premessa. Il Trattato di pace del 10 febbraio 1947, ratificato dall'Italia in virtù della legge di autorizzazione 02 agosto 1947 n. 811, non solo ha stabilito gli attuali confini del territorio italiano, con rinuncia a ogni diritto e titolo sui possedimenti territoriali in Africa ${ }^{132}$, ma ha anche previsto l'istituzione del c.d. Territorio libero di Trieste (sigla TLT), fissando le rispettive frontiere sia sul versante italiano, sia su quello jugoslavo. Ma il Territorio non è mai stato costituito, essendo rimasto diviso in due zone, $\mathrm{A}$ e B, la seconda delle quali è fin da subito ricaduta sotto l'amministrazione della Jugoslavia, mentre la prima, dopo essere stata amministrata dalle autorità militari del Regno Unito e degli Stati Uniti d'America, è passata all'Italia a seguito del Memorandum d'intesa firmato a Londra il 05 ottobre 1954. Se alcuni ${ }^{133}$ ritenevano che, non essendo mai sorto un Territorio libero di

${ }^{131}$ Cfr. A. Favaro, «Parere su "l'indizione della consultazione referendaria...», op. cit., p. 6.

132 Art. 23 del Trattato di Pace.

133 Cfr. A. E. Cammarata, «All'Italia la sovranità su Trieste», Foro it., vol. IV (1950), p. 1. L'autore, in particolare, sosteneva la non cessazione della sovranità italiana in ragione della mancata nomina del Governatore del TLT, quale condizione per l'effettiva costituzione del nuovo ente. A sostegno della tesi di Cammarata va ricordata Corte cass., sez. I, sentenza 09 ottobre 1953 n. 8288 in cui si afferma che «nella dinamica del Trattato (di Pace del 1947) la formazione del nuovo Stato (il Territorio Libero di Trieste) doveva funzionare come necessario presupposto — conditio iuris — della cessazione della sovranità italiana su 
Trieste, l'Italia non avesse mai perso la propria sovranità, di contro la teoria predominante ${ }^{134}$, pur considerando l'insussistenza di uno Stato definibile come Territorio Libero di Trieste, lo ha ritenuto assoggettato a un regime di occupazione militare senza essere nel frattempo soggetto ad alcuna sovranità statale. Un'occupazione la cui potestà era limitata dagli obblighi, impliciti nel Trattato, di non appropriarsi del territorio e di non esercitare la potestà di governo nel senso di pregiudicare la futura sistemazione del territorio stesso. Sebbene l'Italia, l'ha lasciato intendere il giudice costituzionale nella sentenza 23 giugno 1964 n. 53, sentisse il problema del destino della zona $\mathrm{B}$, di una parte cioè «di territorio nazionale sul quale intendeva conservare e riaffermare i suoi diritti» ${ }^{135}$, quando il 01 gennaio 1948 la Costituzione repubblicana è entrata in vigore, essa ha tramutato o meglio «fotografato»gli elementi di fatto del fenomeno storico del territorio esistenti in quel momento, che non comprendevano ancora la zona A, in elementi della fattispecie giuridica ${ }^{136}$. Il Trattato di Osimo del 1975 non è intervenuto ad alterare in senso riduttivo la configurazione territoriale italiana presupposta dalla Carta, ma semmai si è limitato a confermare sul piano formale una situazione oramai consolidata, fissando il confine tra i due Stati in coincidenza con la linea di demarcazione fra l'ex zona A, ritornata all'Italia dopo il Memorandum di Londra del 1954, e l'ex zona $\mathrm{B}^{137}$. Anzi, si potrebbe sostenere che vi sia stato un incremento di territorio per l'Italia, dal momento che alla data di entrata in vigore della Costituzione repubblicana mancava ancora Trieste.

Premesso questo, a me pare che un conto sia il principio di integrità territoriale di cui all'art. 5 della Costituzione per il quale, come insegnava EsPosito, è illegale «ogni attività che entro lo Stato tenda alla divisione della Repubblica italiana in due o più Stati, o alla separazione di una o più parti d'Italia dallo Stato» ${ }^{138}$, un altro l'art. 80 della Carta che presuppone il contesto internazionale, riferendosi a quegli atti di natura pattizia volti a determinare una ridefinizione dei confini territoriali o anche del mare ter-

Trieste». La tesi è stata recentemente sostenuta anche da L. Azzano CantarutTi, L'autodeterminazione..., op. cit.

${ }_{134}$ Cfr. M. Udina, «Aspetti giuridici della questione di Trieste», Rassegna giuliana, 1955, p. 165.

135 Si veda il cons. in dir. della sent. n. 53/1964 Corte cost.

${ }^{136}$ In questo senso P. BIsCARETTI DI RufFiA, voce «Territorio dello Stato», in Enc. dir., vol. XLIV, Milano, Giuffrè, 1992, e F. Cuocolo, Istituzioni di Diritto Pubblico, Milano, Giuffrè, 2003, p. 71.

${ }_{137}$ Così L. Paladin, Diritto Costituzionale, Padova, CEDAM, 1998, p. 113.

138 Cfr. C. Esposito, La Costituzione italiana, Padova, CEDAM, 1954, p. 73. 
ritoriale a seguito principalmente di una guerra (Trattati di pace) o di una conquista territoriale di altro tipo. Del resto, solo la guerra è fattore costitutore di nuove sovranità ${ }^{139}$. Il fatto bellico, sia che prenda corpo fuori e lontano dai confini nazionali, sia che abbia luogo entro quest'ultimi, «possiede potenzialmente una carica dirompente nei riguardi della struttura costitutiva dell'ordinamento, suscettibile di essere profondamente alterata, malgrado il fatto in parola trovi (e debba per Costituzione trovare) la propria giustificazione, la causa come il fine, nell'intento di preservare l'identità-integrità dell'ordinamento» ${ }^{140}$. Questo non significa che lo Stato non possa concludere accordi con altri Stati che importino variazioni di territorio, ma si tratterebbe di un fenomeno inquadrabile nella successione tra Stati che escluderebbe qualunque aspirazione indipendentista di Regioni o di altre parti del territorio italiano.

\section{BIBLIOGRAFIA}

AAVV, Commento dello Statuto della Regione del Veneto, a cura di L. BENVEnUtI, G. Piperata e L. Vandelli, Venezia, Cafoscarina, 2012.

AAVV, Veneto. L'autonomia statutaria, a cura di M. MALO, Torino, Giappichelli, 2012.

AAVV, Il principio di autodeterminazione dei popoli alla prova del nuovo millennio, a cura di M. Distefano, Padova, CEDAM, 2014.

AinIs, M., «Il Veneto viene prima dell'Italia. Lo slogan che diventa Statuto», Corriere della Sera, 23 di ottobre di 2011.

AмоrтH, A., La Costituzione italiana. Commento sistematico, Milano, Giuffrè, 1948.

Antonini, L., «Una terra, un popolo, uno Statuto», Il Diritto della Regione, n. 3 (2011).

Arangio-Ruiz, G., voce «Autodeterminazione (diritto dei popoli alla)», in Enc. giur. Treccani, vol. IV, Roma, 1988.

AzZano Cantarutti, L., «Elaborato per la commissione giuridica risoluzione 44», plebiscito2013.eu, pp. 8-13.

- L'autodeterminazione del popolo veneto tra diritto internazionale e diritto interno, Lezione tenuta in data 12 maggio 2016 presso il Campus universitario Ciels, sede di Milano, paper.

BALBONI, E., «Il referendum consultivo nello Stato-comunità: perché vietarlo?», Le Regioni, n. 1-2 (2001), pp. 216-224.

${ }_{139}$ Cfr. G. DE VergotTinI, Guerra e Costituzione. Nuovi conflitti e sfide alla democrazia, Bologna, Il Mulino, 2004, pp. 159-166.

${ }_{140}$ Cfr. A. Ruggeri, «Le guerre, la teoria del potere costituente e il bilanciamento tra valori costituzionali», Riv. dir. cost. (2005), p. 4. 
Bartole, S., «Riforme federali e consultazioni referendarie regionali: un abbinamento discutibile», Giur. Cost., n. 6 (2000), pp. 3819 e ss.

- Interpretazioni e trasformazioni della Costituzione repubblicana, Bologna, Il Mulino, 2004.

- «La Corte costituzionale "chiude" al federalismo dal basso», Giur. Cost., n. 6 (2007), pp. 4039 e ss.

- «Pretese venete di secessione e storica questione catalana, convergenze e divergenze fra Corte costituzionale italiana e Tribunale costituzionale spagnolo, anche con ripensamenti della giurisprudenza della prima», Giur. Cost., n. 3 (2015), pp. 393 e ss.

Benvenuti, L., «Lo Statuto del Veneto, e oltre», Il Diritto della Regione, n. 3 (2011). Berti, G., «Art. 5 Cost.», in G. Branca (a cura di), Commentario della Costituzione, vol. I, Bologna, Il Mulino, 1975.

Bertolissi, M., «Le iniziative della Regione Veneto per una maggiore autonomia e/o indipendenza», plebiscito2013.eu.

Bifulco, R., «Art. 5 Cost.», in R. Bifulco, A. Celotto e M. Olivetti (a cura di), Commentario alla Costituzione, t. I, Torino, Giappichelli, 2006.

Biscaretti di Ruffia, P., voce «Nazione», in Nov. Dig. It., vol. XI, Torino, UTET, 1965.

— voce «Territorio dello Stato», in Enc. dir., vol. XLIV, Milano, Giuffrè, 1992.

BuzzaCCHI, C., Uniformità e differenziazione nel sistema delle autonomie, Milano, Giuffrè, 2003.

Cammarata, A. E., «All'Italia la sovranità su Trieste», Foro it., vol. IV (1950).

Cammelli, M., voce «Autogoverno», in N. Bobbio, N. Matteucci e G. Pasquino (dirs.), Dizionario di politica, Milano, Giuffrè, 1990.

- «Amministrazione e (interpreti) davanti al nuovo Titolo V della Costituzione», Le Regioni, n. 6 (2001), pp. 1273-1304.

Caravita Di ToritTo, B., «Il tabù della sovranità e gli "istituti tipici di ordinamenti statuali di tipo federale in radice incompatibili con il grado di autonomia regionale attualmente assicurato nel nostro ordinamento costituzionale”», federalismi.it, n. 22 (2007).

— «Federalismi, federalismo europeo, federalismo fiscale», federalismi.it, n. 9 (2011).

Caretti, P., «La "sovranità" regionale come illusorio succedaneo di una "specialità” perduta: in margine alla sent. della Corte costituzionale n. 365/2007», Le Regioni, n. 1 (2008), pp. 219-226.

Carnevale, P., «Unità della Repubblica ed unità del diritto oggettivo. Notazioni introduttive sulla Costituzione come fattore unitario nelle dinamiche della produzione normativa», federalismi.it, n. 1 (2015).

Cassese, A., «Art. 10 Cost.», in G. Branca (a cura di), Commentario della Costituzione, Bologna, Il Mulino, 1975.

- Self-Determination of Peoples. A Legal Reappraisal, Cambridge, Cambridge University Press, 1995. 
Chessa, O., «La resurrezione della sovranità statale nella sent. n. 365/2007», Le Regioni, n. 1 (2008), pp. 227 e ss.

Colaluca, C., voce «Referendum regionali», in M. Malo e P. SAntinello (a cura di), Veneto. Voci per un dizionario giuridico, Torino, Giappichelli, 2015.

Conforti, B., Diritto Internazionale, Napoli, Jovene, 2015.

Conte, F., «La Corte costituzionale sui referendum per l'autonomia e l'indipendenza del Veneto. Non c'è due senza tre. Anche se...», Quad. Cost., n. 3 (2015), pp. 759-761.

Craven, M., «The European Community Commission Arbitration on Yugoslavia», in British Yearbook of International Law, Oxford, Oxford University Press, 1996.

Crawford, J., «The Rights of Peoples: "Peoples" or "Governments"», in J. CRAwFord (a cura di), The Rights of Peoples, Oxford, Clarendon Press, 1988.

CRISAFulli, V., La sovranità popolare nella Costituzione italiana, in Scritti giuridici in memoria di V. E. Orlando, Padova, CEDAM, 1957.

- Stato e popolo nella Costituzione italiana, in Studi per il primo decennale della Costituzione, vol. II, Milano, Giuffrè, 1958.

- Lezioni di Diritto Costituzionale. Introduzione al Diritto Costituzionale italiano, vol. I, Padova, CEDAM, 1970.

Crosa, E., «Il principio della sovranità dello Stato nel diritto italiano», Arch. giur., s. IV, vol. XXV (1933), pp. 145-171.

Cuocolo, F., «Parlamento nazionale e "parlamenti” regionali», Giur. Cost., n. 2 (2002), pp. 873-878.

- Istituzioni di Diritto Pubblico, Milano, Giuffrè, 2003.

D'AloiA, A., «Introduzione. I diritti come immagini in movimento: tra norma e cultura costituzionale», in A. D’Aloia (a cura di), Diritti e Costituzione. Profili evolutivi e dimensioni inedite, Milano, Giuffrè, 2003.

D’AtenA, A., «La vicenda del regionalismo italiano ed i problemi della transizione al federalismo», in A. D'AtenA (a cura di), Federalismo e regionalismo in Europa, Milano, Giuffrè, 1994.

Del Vecchio, G., Lo Stato moderno ed i suoi problemi, Torino, Giappichelli, 1967.

Delledonne, G., «La Corte costituzionale si pronuncia sulla "lingua piemontese": fra tutela delle minoranze linguistiche e incerti limiti di un "costituzionalismo regionale”», Le Regioni, n. 4 (2011), pp. 668-680.

De Martin, G. C., «L'amministrazione pubblica e la Costituzione», astrid-online. it, maggio 2006.

De Vergottini, G., Guerra e Costituzione. Nuovi conflitti e sfide alla democrazia, Bologna, Il Mulino, 2004.

Dickman, R., «Popolo e popolazione nella Costituzione e negli Statuti», federalismi.it, n. 22 (2004).

Dogliani, M., e Cassella, F., «La "solitudine” del Parlamento nella decisione sulla forma dell'unità nazionale», Le Regioni, n. 5 (1993), pp. 1304-1316.

Donati, D., Elementi di diritto costituzionale, Padova, CEDAM, 1932. 
Esposito, C., «Autonomie locali e decentramento amministrativo nell'art. 5 della Costituzione», in La Costituzione italiana. Saggi, Padova, CEDAM, 1954.

- «Commento all'art. 1 della Costituzione», in La Costituzione italiana. Saggi, Padova, CEDAM, 1954.

- La Costituzione italiana, Padova, CEDAM, 1954.

Faraguna, P., «La sentenza n. 238 del 2014: i controlimiti in azione», Quad. Cost., n. 4 (2014), pp. 899-901.

FAVAro, A., «Parere su "l'indizione della consultazione referendaria (...) al fine di accertare la volontà del Popolo Veneto in ordine alla propria autodeterminazione" (Risoluzione n. 44/2012 - Deliberazione n. 145 del Consiglio regionale del Veneto)», plebiscito2013.eu, pp. 2-3.

Ferraiuolo, G., «Due referendum non comparabili», Quad. Cost., n. 3 (2014), pp. 703-706.

- «La Corte costituzionale in tema di referendum consultivi regionali e processo politico: una esile linea argomentativa per un esito (in parte) prevedibile», federalismi.it, n. 20 (2015).

FERRARA, G., «Alcune osservazioni su popolo, Stato e sovranità nella Costituzione italiana», Rass. Dir. Pubbl., n. 2 (1965), pp. 269-294.

Fioravanti, M., «Potere costituente e Diritto Pubblico», in P. Pombeni (a cura di), Potere costituente e riforme costituzionali, Bologna, Il Mulino, 1992.

Frosini, T. E., «Costituzione, autodeterminazione, secessione», Rivista AIC, n. 1 (2015).

GatTI, R., Il popolo dei moderni. Breve saggio su una finzione, Brescia, La Scuola, 2014.

GradONI, L., «La sentenza n. 238/2014 della Corte costituzionale: Corte costituzionale italiana "controvento" sull'immunità giurisdizionale degli Stati stranieri?», Quad. Cost., n. 4 (2014), pp. 905-908.

Grasso, P. G., Il potere costituente, Torino, Giappichelli, 2006.

Guarino, G., Lezioni di diritto pubblico, vol. I, Milano, Giuffrè, 1967.

Herrero de Miñón, M., Memorias de estío, Madrid, Temas de Hoy, 1993.

LatTanzi, F., «Autodeterminazione dei popoli», in Digesto delle Discipline Pubblicistiche, vol. II, Torino, UTET, 1987, pp. 4 e ss.

LEONE, S., «La sentenza n. 238/2014: una soluzione preordinata ad accentrare il sindacato sulle consuetudini internazionali», Quad. Cost., n. 4 (2014), pp. 902-904.

LEWIS GETI, P., «Federalismo linguistico, tutela delle minoranze ed unità nazionale. Un nemis a l'é tròp e sent amis a basto nen. Nota a Corte cost. n. 170/2010», Rivista AIC, n. 4 (2010).

Lupo, N., «Dalla Corte costituzionale uno "stop” (definitivo) ai Parlamenti regionali. Nota a Corte cost. n. 106/2002», amministrazioneincammino.it, n. 10 (2002).

Mancini, S., «Ai confini del diritto: una teoria democratica della secessione», Percorsi Costituzionali, n. 3 (2014), pp. 623-637. 
Marcelli, F., «Il principio di autodeterminazione dei popoli nell'epoca del dominio della finanza», in M. Distefano (a cura di), Il principio di autodeterminazione dei popoli alla prova del nuovo millennio, Padova, CEDAM, 2014.

Milano, E., «Il parere consultivo della Corte internazionale di giustizia sulla dichiarazione di indipendenza del Kosovo: qualche istruzione per l'uso», sidiisil.org, 2010.

— «L'autodeterminazione nei Balcani: soluzioni ad hoc per casi sui generis o consolidamento del principio nel contesto post-coloniale?», in M. Distefano (a cura di), Il principio di autodeterminazione dei popoli alla prova del nuovo millennio, Padova, CEDAM, 2014.

- voce «Autodeterminazione dei popoli», Diritto online. Approfondimenti Enciclopedici. Diritto Internazionale e Comparato, 2014, pp. 1-8.

Mocellin, A., «In merito al referendum per l'indipendenza del Veneto», leggioggi.it, 17 aprile 2015 .

Modugno, F., «Unità-indivisibilità della Repubblica e principio di autodeterminazione dei popoli (Riflessioni sull'ammissibilità-ricevibilità di un disegno di legge costituzionale comportante revisione degli artt. 5 e 132 Cost.)», in A. PACE (a cura di), Studi in onore di Leopoldo Elia, vol. II, Milano, Giuffrè, 1999.

— «Unità e indivisibilità della Repubblica come principio», in P. BIANCHI e C. Latini (a cura di), Costruire l'Italia. Dimensione storica e percorsi giuridici del principio di unità, Napoli, Jovene, 2013.

Morrone, A., «Lo Statuto regionale, dopo le riforme», in A. Cariola, E. CastoriNA e A. Ciancio (a cura di), Studi in onore di Luigi Arcidiacono, Torino, Giappichelli, 2010.

— «Postfazione: a proposito del carattere "veneto" dello Statuto regionale», in F. Benvenuti, G. Piperata e L. Vandelli (a cura di), Commento allo Statuto della Regione del Veneto, Venezia, Cafoscarina, 2012, pp. 1-7, nonché come «Avanti popolo...regionale!», Quad. Cost., n. 3 (2012), pp. 615-618.

Mortati, C., Istituzioni di diritto pubblico, vol. I, Padova, CEDAM, 1969.

Nocilla, D., voce «Popolo (dir. cost.)», in Enc. dir., vol. XXXIV, Milano, Giuffrè, 1985.

Orlando, V. E., Principi di diritto costituzionale, Firenze, G. Barbèra, 1917.

Paladin, L., «Valori nazionali e principio di unità della Repubblica nella Costituzione italiana», in A. PACE (a cura di), Studi in onore di Mazziotti, Padova, CEDAM, 1995.

- Le fonti del diritto italiano, Bologna, Il Mulino, 1996.

- Diritto Costituzionale, Padova, CEDAM, 1998.

- Saggi di Storia costituzionale, a cura di S. Bartole, Bologna, Il Mulino, 2008.

Palermo, F., e Woelk, J., Diritto Costituzionale comparato dei gruppi e delle minoranze, Padova, CEDAM, 2008.

Palmisano, G., voce «Autodeterminazione dei popoli», Enc. dir., Annali, vol. V, Milano, Giuffrè, 2012. 
PARIOTTI, E., I diritti umani: concetto, teoria, evoluzione, Padova, CEDAM, 2013.

Perassi, T., «La Costituzione italiana e l'ordinamento internazionale», in Scritti giuridici, Milano, Giuffrè, 1958.

Pertile, M., «Il parere sul Kosovo e l'autodeterminazione assente: quando la parsimonia non è una virtù», in L. GRADONI e E. Milano (a cura di), Il parere della Corte internazionale di giustizia sulla dichiarazione di indipendenza del Kosovo: un'analisi critica, Padova, CEDAM, 2011.

Picone, P., «Le reazioni collettive ad un illecito erga omnes in assenza di uno Stato individualmente leso», in P. PiCONE (a cura di), Comunità internazionale e obblighi erga omnes, Napoli, Jovene, 2013.

Pietrangelo, M., «Qualche riflessione sui metodi della consultazione popolare al tempo di Internet, a margine dei referendum consultivi veneti su indipendenza ed autonomia», federalismi.it, n. 1 (2015).

Piperata, G., «Art. 2 Statuto della Regione del Veneto», in L. Benvenuti, G. PiPeRATA e L. VANDElli (a cura di), Commento dello Statuto della Regione del Veneto, Venezia, Cafoscarina, 2012.

PIstorio, G., «Il principio di unità ed indivisibilità della Repubblica nella giurisprudenza costituzionale», federalismi.it, n. 1 (2015).

Pubusa, A., Sovranità popolare e autonomie locali nell'ordinamento costituzionale italiano, Milano, Giuffrè, 1983.

RAIC, D., Statehood and the Law of Self-Determination, The Hague, Kluwer Law International, 2002.

Rangeon, F., «Intérêt général et démocratie», Cabiers de philosophie politique et juridique de l'Université de Caen, n. 2 (1982).

Romboli, R., «Problemi interpretativi della nozione giuridica di popolo», Riv. trim. dir. pubbl., vol. 34 (1984), pp. 159 e ss.

- voce «Popolo», Enc. giur. Treccani, vol. XXIII, Roma, 1991.

RonziTTI, N., «L'adattamento dell'ordinamento italiano alle norme imperative di diritto internazionale», Rass. parlam., n. 2 (2002), pp. 503 e ss.

- Introduzione al Diritto Internazionale, Torino, Giappichelli, 2016.

Ruggeri, A., «Ancora in tema di referendum regionali consultivi e di teoria delle fonti», Le Regioni, n. 1-2 (2001), pp. 224 e ss.

- «Le guerre, la teoria del potere costituente e il bilanciamento tra valori costituzionali», Riv. dir. cost. (2005), pp. 3-26.

— «La Corte aziona l'arma dei "controlimiti" e, facendo un uso alquanto singolare delle categorie processuali, sbarra le porte all'ingresso in ambito interno di norma internazionale consuetudinaria», in «Itinerari» di una ricerca sul sistema delle fonti. Studi dell'anno 2014, vol. XVIII, Torino, Giappichelli, 2015.

RUPERTO, C., «La legalità costituzionale», associazionedeicostituzionalisti.it, 2001.

SAlERNO, G. M., «Principio di unità e ragionevole decentramento della Repubblica», in M. Bertolissi (a cura di), Riforme. Opinioni a confronto, Napoli, Jovene, 2015. 
SAlomoni, F., «Legalità (costituzionale) e forma di Stato: aspetti teorici e profili partici di due concetti apparentemente in crisi», Riv. dir. cost. (2004), pp. 108-152.

Serges, G., «Autodeterminazione, diritto a decidere, indipendenza, sovranità (notazioni a margine della legge regionale del Veneto, n. 16 del 2014)», federalismi.it, n. 1 (2015).

Tosato, E., Persona, Società intermedie e Stato. Saggi, Milano, Giuffrè, 1989.

Udina, M., «Aspetti giuridici della questione di Trieste», Rassegna giuliana, 1955.

VARI, F., «La sentenza n. 238 del 2014: ancora sulla responsabilità degli Stati», Quad. Cost., n. 1 (2015), pp. 166-168.

Veronesi, P., «Il concetto costituzionale di «unità nazionale» e le sue implicazioni», in A. PugiotTo (a cura di), Per una consapevole cultura costituzionale. Lezioni magistrali, Napoli, Jovene, 2013.

VIOLI, F., «Autodeterminazione dei popoli e nuove forme di colonialismo», in M. DisteFAno (a cura di), L'autodeterminazione dei popoli alla prova del nuovo millennio, Padova, CEDAM, 2014, pp. 105-136.

VIPIANA, P., «Le ripercussioni della crisi economica sull'assetto delle autonomie in Italia: un ritorno all'accentramento», in P. VIPIANA (a cura di), Tendenze centripete e centrifughe negli ordinamenti statali dell'Europa in crisi, Torino, Giappichelli, 2014, pp. 20 e ss.

Zagrebelski, G., Il sistema delle fonti del diritto, Torino, Giappichelli, 1984. 